TITLE:

\title{
Time-domain ab initio modeling of excitation dynamics in quantum dots
}

$\operatorname{AUTHOR}(\mathrm{S}):$

Neukirch, Amanda J.; Hyeon-Deuk, Kim; Prezhdo, Oleg V.

\section{CITATION:}

Neukirch, Amanda J....[et al]. Time-domain ab initio modeling of excitation dynamics in quantum dots. Coordination Chemistry Reviews 2014, 263-264: 161-181

ISSUE DATE:

2014-03

URL:

http://hdl.handle.net/2433/193468

\section{RIGHT:}

(c) 2013 Elsevier B.V.; この論文は出版社版でありません。引用の際には 出版社版をご確認ご利用ください。; This is not the published version. Please cite only the published version. 


\title{
A Time-Domain Ab Initio View of Charge and Exciton Dynamics in Semiconductor Quantum Dots
}

Amanda J. Neukirch, ${ }^{1}$ Kim Hyeon-Deuk ${ }^{2}$, and Oleg V. Prezhdo ${ }^{1,3}$

\author{
University of Rochester ${ }^{1}$ \\ Department of Physics and Astronomy \\ Bausch \& Lomb Hall \\ P.O. Box 270171 \\ 500 Wilson Boulevard \\ Rochester, NY 14627-0171 \\ Kyoto University ${ }^{2}$ \\ Department of Chemistry \\ Kyoto University \\ Kyoto, 606-8502, Japan \\ University of Rochester ${ }^{3}$ \\ Department of Chemistry \\ RC Box 270216 \\ Rochester, NY 14627-0216 \\ Fax: (585) 276-0205 \\ Phone: (585) 273-5687
}

Email: oleg.prezhdo@ rochester.edu 


\begin{abstract}
This review discusses using ab initio time-dependent density functional theory and the fewest switches surface hopping algorithm to perform non-adiabatic molecular dynamics in order to model the photoexcited dynamics of electrons and holes in semiconductor quantum dots (QD). The contained methods create an explicit time-domain representation of photoinduced processes such as multiple exciton generation, fission, and recombination (MEG, MEF, and MER). These nonequilibrium phenomena control the optical properties and photoexcited dynamics of QDs. Our approach can account for QD size and shape as well as defects, shells, surface ligands, and charge trap states. Each of these variations significantly changes the properties of photoexcited QDs. For example, the simulations show that high frequency phonon modes from ligands on a QD surface play a crucial role in MEG, MEF, MER, and electron-phonon relaxation. The insights reported in this review provide new ways of controlling photo-induced processes in semiconductor QDs; leading to design principles that will increase the efficiencies of photovoltaic devices.
\end{abstract}


Table of Contents

1. Introduction

2. Theoretical Approaches

2.1.Hartree Fock Method

2.2. SAC-CI

2.3. Density Functional Theory

2.4. Time-domain density functional theory

2.5. Nonadiabatic Molecular Dynamics and Fewest switches surface hopping

2.6.Optical Response Functions and Dephasing Times

2.7.MEG

3. MEG

3.1.Direct Photoexcitaiton Mechanism

3.1.1. Direct MEG in Small Clusters with SACCI

3.1.2. MEG with dopants defects and charges

3.2.Impact Ionization Mechanism

3.2.1 Single and Multiple Exciton Density of States

3.2.2 Real-Time Dynamics of Multiple Exciton Generation

3.2.3 Real-Time Interplay between the Multiple Exciton Generation and

Recombination

3.3.Phonon-induced Dephasing Mechanism

3.3.1 Luminescence, Multiple Exciton Generation, and Multiple Exciton Fission

3.3.2 Temparature Dependence

3.3.3 Size Dependence

3.3.4 Various Electronic States - Bulk, Surface and Plasmon States -

4. Electron Phonon Relaxation

4.1.PbSe

4.2.Plasmons

4.3.Temperature Dependence

4.4.Phonon Bottlneck/dephasing

4.5. Core/shell photoluminescence 


\subsection{Ligands}




\section{$1 \quad$ Introduction}

Quantum dots are nanoscale clusters of bulk material whose electronic excitations are confined in all three spatial dimensions. Every material has its own exciton Bohr radius, which is the physical size of an excitation in that particular material. When the dimensions of a nanocrystal reach this characteristic length, it starts to exhibit the effects of quantum confinement much like a particle in a box. This allows for the properties of the QD to be tuned continuously by changing the cluster's size and shape. Once the system reaches the subnanometer scale it begins to behave like a molecule; its structure varies from bulk and its properties change discontinuously with cluster size.

The excited state dynamics in semiconducting ${ }^{1}$ and metallic ${ }^{2}$ QDs is complex. High absorption cross sections, decreased electron-phonon relaxation rates, ${ }^{3,4}$ and generation of multiple electron-(ME) hole pairs ${ }^{5-7}$ make QDs excellent photovoltaic materials since these features all provide opportunities to utilize the photon energy in excess of the band gap. ${ }^{3,8}$ Electron-hole and charge-phonon interactions have both fundamental and practical importance, since both interactions contribute to the overall efficiency of a photovoltaic device. Charge transfer processes are limited by phonon-induced dephasing of spin and electron states. Inelastic scattering is responsible for energy loss during charge tunneling though QDs.

Relaxation and dephasing are two related but distinct phenomena that result from electronphonon interactions. Relaxation is an inelastic process that leads towards energy losses, decreasing the solar cell voltage. Electron-phonon dephasing is an elastic process, and therefore conserves electronic energy. Dephasing determines the homogenous linewidths in optical spectra. It destroys the coherence between electronic states transforming coherent superpositions into uncorrelated ensembles. The loss of correlation in coherent superpositions of multiple excitons (MEs) contributes to MEF. Phonon induced dephasing of coherent superpositions of single and ME states is an essential component of the mechanism of ME generation (MEG).

MEG, also known as carrier multiplication (CM), is the process in which a single high-energy photon is absorbed and creates two or more electron-hole pairs. This phenomena provides potential for increasing photovoltaic device efficiencies. MEG happens in bulk, but is typically more efficient in QDs. This is because in bulk materials crystal momentum, a pseudo-momentum associated with electrons in a lattice, needs to be conserved. This requirement causes the energy needed to form a biexciton to be more than simply two times the bandgap. A lack of translational symmetry in QDs precludes/voids the need to 
conserve crystal momentum.(Zunger APL 84 (13) 2004). Also Coulomb interaction between electrons and holes is enhanced due to the closer proximity of charge carriers. (NL 8(10) 31742008 ).

This review presents a cutting edge ab initio description of the time-dependent dynamics of photoexcited states in semiconductor QDs. These $a b$ initio methods are used to study excited state composition, evolution and relaxation, as well as electron-phonon dephasing, all with an eye towards the incorporation of QDs in solar cells. The time-dependent atomistic nature of er-methods provide powerful tools for studying the role of surface ligands, dopants, defects, unsaturated bonds, size, shape and other realistic aspects of QDs. The described simulations give a comprehensive perspective on the elastic and inelastic scattering dynamics of photoexcited charge carriers in nanoscale materials, offering insights into the mechanisms involved in solar energy harvesting. For further reading on the work featured in this chapter, see references. ${ }^{8-10}$

\section{Theoretical Approaches}

We use a wide array of ab initio methods to study the photoexcited dyamics in semiconductor quantum dots. The simulations described in this review treat many-body interactions in the electronic degrees of freedom for either fixed nuclear coordinates or nuclei evolving classically. Symmetry adapted cluster (SAC) theory with configuration interactions (CI) is used to describe photoinduced multiple exciton generation for fixed nulcei. Exciton dynamics, including impact ionization and electron-phonon relaxation, are modeled using time-dependent density functional theory (TDDFT). Here excitons are coupled to phonon motions with nonadiabatic molecular dynamics (NAMD) methods including the fewest switches surface hopping (FSSH) algorithm. The optical response formalism is used to obtain information on electron-phonon dephasing which has an influence in the exciton formation as well as relaxation.

\subsection{Hartree-Fock Method}

Even the smallest quantum dots consist of hundreds of atoms leading to systems with tens of thousands of electrons. For example, a $\mathrm{Pb}_{68} \mathrm{Se}_{68} \mathrm{QD}$ with diameter of only $2 \mathrm{~nm}$ already has 8,000 electron. Solving the exact many-body wavefunction for this system would be impossible. Fortunately physically motivated approximations have been shown to bring tractability to the many body problem. One of the simplest and earliest methods developed is the Hartree-Fock (HF) method. HF theory was developed to solve the electronic Schrödinger equation for give set of nuclear coordinates. The basic assumption behind the HF method is that the many-electron wave function can be written as a product of 
one-electron orbitals. An individual electron in the system feels an electrostatic field from the central potential of the nuclei and other electrons, Fig. 1. HF is a self-consistent theory in that the electron orbitals are determined by the mean-field, which depends on the orbitals. HF includes the Pauli Exclusion Principle by not allowing two identical electrons to occupy the same location through its exchange interaction; however no electron correlation is incorporated.

\subsection{Incorporation of Electron Correlation in Hartee-Fock with Configuration Interaction}

The non-interacting single-particle picture is provided by the Hartree-Fock approximation, which excludes electron-correlation effects. Characterizing the excited states in the semiconductor QDs as single or MEs requires a proper single-particle description and a rigorous account of the electron-hole Coulomb interaction. This correlation can be systematically added to HF using symmetry adapted cluster (SAC) theory with configuration interactions (CI). Including explicit electron correlations allows for the exact many body wave functions to be obtained, although at such a large computational expense as to only allow for the calculation of small clusters. Configurations of multi-body correlations, Figure 1, represent multiple excitons, whose properties are particularly important for QD photochemistry. SAC-CI allows for excited states to be quantified as single or MEs because of its ability to explicitly treat multiply excited electrons and holes. For example, the SAC-CI used below explicitly includes electron correlation through cluster expansion of the ground state wave function (WF).

$$
\left|\Psi^{S A C}\right\rangle=\exp \left(\sum_{I=1}^{M} C_{I} \hat{S}_{I}\right)\left|\Phi_{0}\right\rangle=\left(1+\sum_{I} C_{I} \hat{S}_{I}+\frac{1}{2} \sum_{I, J} C_{I} C_{J} \hat{S}_{I} \hat{S}_{J}+\cdots\right)\left|\Phi_{0}\right\rangle
$$

Here, $\left|\Phi_{0}\right\rangle$ represents the $\mathrm{HF} \mathrm{WF}, \hat{S}_{I}$ are symmetry adapted excitation operators, and $C_{I}$ are variable coefficients. The excited state WFs $\left|\Psi^{S A C-C I}\right\rangle$ are calculated from the electron correlated ground state WF $\left|\Psi^{S A C}\right\rangle$

$$
\left|\Psi^{S A C-C I}\right\rangle=\sum_{K=1}^{N} d_{K} R_{K}\left|\Psi^{S A C}\right\rangle,
$$

where $R_{K}$ represents an excitation operator, and $d_{K}$ is the SAC-CI coefficient. A coefficicent is associated with each configuration that represents that configuration's contribution to the electronic structure of the excited state. This allows for the excited states to be classified as either SE or ME.

\subsection{Density Functional Theory}

In contrast to SAC-CI, DFT accounts for electron correlations indirectly. The Hohenberg-Kohn theorem proves that the ground state properties of a many body system are uniquely determined by the electron density, which only depends on three spatial coordinates. Properties of the system, such as Coulomb interactions, are then described through use of functionals of the electron density. Kohn-Sham (KS) DFT ensures that the system of non-interacting particles in an effective potential generates the 
same density as a system of interacting particles. KS DFT is therefore a single-particle representation that accounts for electron correlations, incorporationg electron-hole interactions and excitonic effects. HF can be viewed as a special form of DFT that includes exact Pauli exchange and no electron correlation. KS DFT is computationally inexpensive and works particularly well with extended systems that do not undergo complex chemical changes.

\subsection{Time-Domain Density Functional Theory}

The Runge-Gross theorem shows that the three-dimensional density of a many body quantum system is sufficient to describe the TD response of the system to an external perturbation, including electro-magnetic field and vibrational motions, $R(t)$. This is known as TDDFT, and describes a system's response to external perturbations, such as electromagnetic fields and phonons. Linear response TDDFT is frequently used to evaluate electronic excitation energies. The Full TDDFT employed in the studies discussed in this review explicitly propagates electron density in time. Solutions obtained from timeindependent DFT are used as the starting point for our TDDFT calculations. ${ }^{11}$

The electron density in the time-dependent density functional theory (TDDFT) is written in the KS representation as ${ }^{12}$

$$
\rho(\boldsymbol{r}, t)=\sum_{p=1}^{N_{e}}\left|\varphi_{p}(\boldsymbol{r}, t)\right|^{2}
$$

Where $\mathrm{N}_{\mathrm{e}}$ is the number of electrons and $\varphi_{p}(r, t)$ are single-electron KS orbitals. The evolution of the $\varphi_{p}(r, t)$ is determined by the application of the TD vibrational principle to the KS energy ${ }^{12}$

$E\left\{\varphi_{p}\right\}=\sum_{p=1}^{N_{e}}\left\langle\varphi_{p}|K(\boldsymbol{r})| \varphi_{p}\right\rangle+\sum_{p=1}^{N_{e}}\left\langle\varphi_{p}|V(\boldsymbol{r} ; \boldsymbol{R})| \varphi_{p}\right\rangle+\frac{e^{2}}{2} \iint \frac{\rho\left(\boldsymbol{r}^{\prime}\right) \rho(\boldsymbol{r})}{|\boldsymbol{r}-\boldsymbol{r}|} d^{3} \boldsymbol{r} d^{3} \boldsymbol{r}^{\prime}+E_{x c}\{\rho\},(4)$

which contains the kinetic energy of noninteracting electrons $K(\boldsymbol{r})$, the electron-nuclear attraction $V(\boldsymbol{r} ; \boldsymbol{R})$ which depends on the phonon coordinates $\boldsymbol{R}$, the Coulomb repulsion of density $\rho(\boldsymbol{r}, t)$, and the exchangecorrelation energy functional $E_{x c}$ that accounts for the lingering many-body interactions. Application of the TD (Dirac) variational principle to KS energy states generates a system of coupled equations of motion for single particle KS orbitals (*Gross An. Rev. Phys. Chem. 2004, 55, 427; JCP2004, 121 903; PRB2005, 71, 033201*)

$$
i \hbar \frac{\partial \varphi_{p}(r, t)}{\partial t}=H(r ; \boldsymbol{R})\{\varphi(r, t)\} \varphi_{p}(r, t), \quad p=1, \ldots, N_{e} .
$$

The TDKS equations are coupled, since the Hamiltonian $H$ depends on the overall density, Eq. 2.9, and, hence, all occupied KS orbitals. We solve the TDKS equation by expanding the TDKS orbitals $\varphi_{p}(r, t)$ in the adiabatic KS orbital $\tilde{\varphi}_{k}(r ; \boldsymbol{R})$ basis obtained in the ground state DFT calculation,

$$
\varphi_{p}(r, t)=\sum_{k}^{N_{e}} c_{p k}(t)\left|\tilde{\varphi}_{k}(r ; \boldsymbol{R})\right\rangle
$$


Then, Eq. 5 transforms into the equation for the expansion coefficients,

$$
i \hbar \frac{\partial c_{p k}(t)}{\partial t}=\sum_{m=1}^{N_{e}} c_{p m}(t)\left(\epsilon_{m} \delta_{k m}-i \hbar \boldsymbol{d}_{k m} \cdot \dot{\boldsymbol{R}}\right)
$$

The NA coupling describes the electron-phonon interaction,

$$
\boldsymbol{d}_{k m} \cdot \dot{\boldsymbol{R}}=\left\langle\tilde{\varphi}_{k}(\boldsymbol{r} ; \boldsymbol{R})\left|\nabla_{\boldsymbol{R}}\right| \tilde{\varphi}_{m}(\boldsymbol{r}, \boldsymbol{R})\right\rangle \cdot \dot{\boldsymbol{R}}=\left\langle\tilde{\varphi}_{k}(\boldsymbol{r}, \boldsymbol{R})\left|\frac{\partial}{\partial t}\right| \tilde{\varphi}_{m}(\boldsymbol{r}, \boldsymbol{R})\right\rangle
$$

arises from the dependence of the adiabatic KS orbitals on the phonon coordinates $\boldsymbol{R}(\boldsymbol{t}) .{ }^{13}$ Nuclear trajectories from the ground state are used to sample initial conditions to create ensemble averages for the NA dynamics. NAMD includes transitions between electronic states.

\subsection{Nonadiabatic Molecular Dynamics: fewest switches surface hopping}

FSSH prescribes a probability for hopping between electronic states. The probability is explicitly time dependent and is correlated with the nuclear evolution. The probability of hopping between states $k$ and $m$ within the time interval $\Delta t$ depends explicitly on phonon dynamics and equals ${ }^{14}$

$$
g_{k m}(t, \Delta t)=\max \left(0, \frac{b_{k m} \Delta t}{a_{k k}(t)}\right)
$$

where

$$
b_{k m}=-2 \operatorname{Re}\left(a_{k m}^{*} \boldsymbol{d}_{k m} \cdot \dot{\boldsymbol{R}}\right) ; \quad a_{k m}=c_{k} c_{m}^{*}
$$

The coefficients $c_{k}$ and $c_{m}$ evolve according to Eq. 7, $\boldsymbol{d}_{k m} \cdot \dot{\boldsymbol{R}}$ is the NA coupling in Eq. 8. If the calculated $g_{k m}$ is negative, the hopping probability is set to zero; a hop from state $k$ to state $m$ can only occur if the electronic occupation of state $k$ decreases and the occupation of state $m$ increases. This feature of the algorithm minimizes the number of hops and is responsible for the name of the technique "fewest switches". To conserve the total electron nuclear energy after a hop, the original FSSH technique rescales the nuclear velocities along the direction of the electronic component, $\mathbf{d} \mathbf{k m}$, of the NA coupling. ${ }^{13,14}$ If the kinetic energy available to the nuclei along the direction of the NA coupling is insufficient to accommodate an increase in the electronic energy, the hop is rejected. The hop-rejection creates the detailed balance between upward and downward transitions. ${ }^{15}$

In the simplified implementation of FSSH discussed in this review, it is assumed that the energy exchanged between the electronic and vibrational degrees of freedom during a hop are redistributed rapidly among all of the vibrational modes. The velocity rescaling and hop rejection is replaced by 
multiplying the probability (Eq. 9) for transitions upward in energy by the Boltzmann factor. This allows for the use of a ground state nuclear trajectory to determine the TD potential that drives the electron dynamics. This simplification leads to significant improvements in the computational efficiency of FSSH. The FSSH simulations are performed separately for electrons and holes in the basis of singleparticle adiabatic KS orbitals. The single-particle representation is appropriate for studies of QDs since their electronic structure is well represented by the independent electron and hole picture.

It is important to note that by treating nuclei classically, the original FSSH scheme excludes coherence loss that occurs in the electronic subsystem by coupling to quantum vibrations. Decoherence can be neglected if it is slower than the electronic transition. However, in some instances when the decay is slow, decoherence must be explicitly included in the quantum-classical simulations. Decoherence is implanted within TDDFT-FSSH using a simple semiclassical approach(PRL 100, 197402). The expansion coefficients of the KS wave functions are allowed to evolve coherently up to the decoherence time, at this point they are reset to 0 or 1 with the probabilities given by the squares of the coefficients. The decoherence times, or vibrationally-induced dephasing times, are computed using the optical response function formalism.

\subsection{Optical Response Function for Pure Dephasing}

Electron-phonon interactions give rise to two related but qualitatively distinct phenomena: relaxation and dephasing. Compared to the electron-phonon energy relaxation, the electron-phonon dephasing is a more subtle effect. The dephasing is an elastic process that conserves the electronic energy. The pure-dephasing time is associated with fluctuations and uncertainties in the energy levels due to coupling of electrons to phonons in the semiconductor, ligands, and solvent, etc. The fluctuations in the energy levels are best characterized in terms of correlation functions. The unnormalized autocorrelation function (ACF) for a transition of energy $E$ is defined as

$$
C_{u}(t)=\langle\Delta E(t) \Delta E(0)\rangle
$$

where $\Delta E=E-\langle E\rangle$, and the angular brackets denote averaging over a statistical ensemble, in particular, canonical averaging in the present study. The initial value of the unnormalized ACF gives the average fluctuation in the transition energy, $C_{u}(0)=\left\langle\Delta E^{2}(0)\right\rangle$. Dividing $C_{u}(t)$ by $C_{u}(0)$ gives the normalized ACF: 


$$
C(t)=\frac{\langle\Delta E(t) \Delta E(0)\rangle}{\left\langle\Delta E^{2}(0)\right\rangle}
$$

ACFs characterize periodicity and memory of the energy fluctuations. Rapid decay of an ACF indicates short memory and occurs if multiple phonons modes couple to the electronic transition and if these modes are anharmonic.

The Fourier transform of the ACF is known as the influence spectrum:

$$
I(\omega)=\left|\frac{1}{\sqrt{2 \pi}} \int_{-\infty}^{\infty} d t e^{-i \omega t} C(t)\right|^{2}
$$

It identifies the frequencies of the phonon modes that efficiently couple to the electronic subsystem. The strength of the electron-phonon coupling for a particular mode is related to the intensity of the corresponding line in the influence spectrum.

The optical response functions characterizing the dephasing processes for a pair of states that are entangled in a coherent superposition can be obtained directly or via the second order cumulant expansion. The cumulant expansion approximation (Eq. 14), together with the Fourier transform of the ACF, provides additional information about the dephasing process. The cumulant dephasing function is obtained by double integration and exponentiation of the unnormalized ACF, Eq. 11.

$$
D(t)=\exp (-g(t)), g(t)=\frac{1}{\hbar^{2}} \int_{0}^{t} d \tau_{1}^{\tau_{1}} \int_{0} d \tau_{2} C_{u}\left(\tau_{2}\right)
$$

The above expression indicates that rapid dephasing is facilitated by a large fluctuation of the transition energy (i.e. large $C_{u}(0)=\left\langle\Delta E^{2}(0)\right\rangle$ ) rather than by a rapid memory loss of the fluctuation (i.e. rapidly decaying ACF). [NOTE: the rapid decay of $C_{u}(t)$ itself lets $D(t)$ survive even longer since the rapid decay of $\mathrm{Cu}(\mathrm{t})$ leads to smaller $\mathrm{g}(\mathrm{t})$.]

Alternatively, the dephasing function can be computed directly as

$$
D(t)=\exp (i \omega t)\left\langle\exp \left(-\frac{i}{\hbar} \int_{0}^{t} \Delta E(\tau) d \tau\right)\right\rangle
$$

Here, $\omega$ is the thermally averaged transition energy $\langle\Delta E\rangle$ divided by $\hbar$. With the direct expression, it is more difficult to achieve convergence, since it involves averaging of a complex-valued oscillatory 
function, $\exp \left(-\frac{i}{\hbar} \int_{0}^{t} \Delta E(\tau) d \tau\right)$, whose real and imaginary parts change signs. In comparison, the cumulant expression, Eqs. (11) and (14), involves the averaging of a real and pesitively-valued transition energy and its ACF.

\section{Time-dependent density functional theory for MEG and MER}

MEG and MER are known as typical Auger processes. We simulate Auger dynamics involving single and double exciton states coupled to nuclear motions with TDDFT formulated in the adiabatic KS basis (J. Phys. Condes. Matter 32 363201; Nano Lett. 11 1845; ACS Nano 6 1239). In the adiabatic representation, all Coulomb interactions appearing in the electronic Hamiltonian are 'diagonalized out' during the calculations of the adiabatic states, and transitions between different SE and DE states occur due to the NA coupling, as described below.

Our simulation method including the ground, SE, and DE states, $\left|\phi_{g}(\boldsymbol{x} ; \boldsymbol{R})\right\rangle,\left|\phi_{S E}^{i, j}(\boldsymbol{x} ; \boldsymbol{R})\right\rangle$, $\left|\phi_{D E}^{i, j, k, l}(\boldsymbol{x} ; \boldsymbol{R})\right\rangle$, respectiviely, was formulated using second quantization with the ground state as a reference. SEs and DEs are obtained as

$$
\left|\phi_{S E}^{i, j}\right\rangle=\hat{\mathrm{a}}_{i}^{\dagger} \hat{\mathrm{a}}_{j}\left|\phi_{g}\right\rangle, \quad\left|\phi_{D E}^{i, j, k, l}\right\rangle=\hat{\mathrm{a}}_{i}^{\dagger} \hat{\mathrm{a}}_{j} \hat{\mathrm{a}}_{k}^{\dagger} \hat{\mathrm{a}}_{l}\left|\phi_{g}\right\rangle
$$

where the electron creation and annihilation operators, $\hat{\mathrm{a}}_{i}^{\dagger}$ and $\hat{\mathrm{a}}_{j}$, generate and destroy an electron in the $\mathrm{i}^{\text {th }}$ and $\mathrm{j}^{\text {th }}$ adiabatic KS orbitals, respectively. The time-evolving wave function is then expressed by

$$
|\Psi(t)\rangle=C_{g}(t)\left|\phi_{g}\right\rangle+\sum_{i, j} C_{S E}^{i, j}(t)\left|\phi_{S E}^{i, j}\right\rangle+\sum_{i, j, k, l} C_{D E}^{i, j, k, l}(t)\left|\phi_{D E}^{i, j, k, l}\right\rangle
$$

Similarly to Eq. 7, the expansion coefficient appearing in Eq.17, evolves by the first-order differential equations

$$
\begin{aligned}
& i \hbar \frac{\partial C_{X}(t)}{\partial t}=C_{X}(t) E_{X}-i \hbar C_{g}(t) d_{X ; g} \cdot \dot{\boldsymbol{R}}-i \hbar \sum_{i^{\prime}, j^{\prime}} C_{S E}^{i^{\prime}, j^{\prime}}(t) d_{X ; S E, i^{\prime}, j^{\prime}} \cdot \dot{\boldsymbol{R}}- \\
& i \hbar \sum_{i^{\prime}, j^{\prime}, k^{\prime}, l^{\prime}} C_{D E}^{i^{\prime}, j^{\prime}, k^{\prime}, l^{\prime}}(t) d_{X ; D E, i^{\prime}, j^{\prime}, k^{\prime}, l^{\prime}} \cdot \dot{\boldsymbol{R}}
\end{aligned}
$$

where $\mathrm{X}$ and $\mathrm{Y}$ now correspond to either ground, SE, or DE state, $\mathrm{E}_{\mathrm{X}}$ is the state energy, and the NA couplings are defined by

$$
d_{X ; Y} \cdot \dot{\boldsymbol{R}} \equiv\left\langle\phi_{X}\left|\nabla_{R}\right| \phi_{Y}\right\rangle \cdot \dot{\boldsymbol{R}}=\left\langle\phi_{X}\left|\frac{\partial}{\partial t}\right| \phi_{Y}\right\rangle
$$


The atomistic simulation of SE/DE generation and dynamics was performed by directly solving eq. 18 with time-dependent NA couplings and energies. The ground, SE and DE states form the two-particle electronic basis in our method, and each state can transit to another state due to the NA coupling. The energies appear in the diagonal parts of the Hamiltonian, while the NA couplings are embedded in the corresponding off-diagonal components. The simulations are extremely large-scale. Too large for direct numerical simulation; however, the Hamiltonian is sparse, since the NA couplings connect states that differ only in a single electron or hole. Based on this fact, we developed an efficient simulation code that can remove all zero components from the sparse Hamiltonian, and solve eq. 18 using only the extracted non-zero components of the Hamiltonian.

\section{Proposed Mechanisms for Multiple Exciton Generation}

One of the most promising features of QDs for increased efficiency in solar cells is multiexciton generation (MEG). Predicted ${ }^{3}$ several years before discovery, ${ }^{5}$ MEG from high energy photons avoids energy losses associated with electron-phonon relaxation to lower energy levels. MEG has drawn close attention due to its potential for substantial improvement of photovoltaic device efficiencies. ${ }^{3,5,6,8,16-23}$ The $a b$ initio analysis of the electronically excited states in the semiconductor QDs allows for the different proposed mechanisms for the MEG to be critically assessed. ${ }^{3,6,20,24}$ Different proposals echo the rapidly shifting views on MEs and the phonon bottleneck and reflect the variety in the electronic structure of the materials exhibiting MEs. The $a b$ initio electronic structure calculations ${ }^{22}$ discussed below will show two distinct pathways towards MEG, direct photoexcitaiton and auger processes(cite rabani 2010). MEG is intimately related to phonon-induced dephasing. Dephasing destroys coherences between electronic states and converts them into ensembles of uncorrelated states. The loss of electronic coherence occurs by coupling to phonons and, in this case, should be faster than MEG. Finally, no matter how MEs are created they must dissociate into uncorrelated excitons that coexist in the same QD and luminesce independently. This loss of correlation with in the coherent superposition of MEs has been named ME fission (MEF), and can be explained by phonon induced dephasing. (***ADD things talking about Direct Photoexcitaiton and Auger Processes and Metion Figure 2***)

\subsection{Direct Photoexcitation Mechanism}

Schaller et al. 5, 8, 19, 20, 22 proposed that a single absorbed photon generates a bi-exciton instantaneously by a second order perturbative process involving bi-exciton coupling to virtual single exciton states. (*may not want to open with this, technically different description*) Photoexcitation of multi-electron states is forbidden in the independent-particle description. The Coulomb interaction 
between independent electrons and holes couples singly and multiply excited states, generating nonvanishing oscillator strength for the multi-electron excitations.

Configuration interaction (CI) describes an excited state as a superposition of all possible excitations from the Hatree-Fock ground state. Using SAC-CI in the static, many-body picture, photoexcited states of small clusters can be characterized as a superposition of electronic configurations in a molecular orbital basis. The band gap is taken to be difference between the ground state and the first excited state. Each electronic configuration is assigned CI coefficient that denotes that configuration's significance or contribution to the electronic structure of the excited state. Large coefficients for singly excited configurations indicate that the excited state corresponds to the generation of a single exciton. Large coefficients for double excited configurations indicate nontrivial contributions from bi-excitonic excitation. Throughout this section SAC-CI is implemented within the Gaussian 03 computational package using the B3LYP functional. The LANL2 relativistic effective core potentials were used for core electrons of all atoms, and the basis set employed for the valence electrons was the corresponding LANL2DZ basis set. MEG thresholds for lead selenide, cadmium selenide, and silicon are determined for ideal, charged, and nonstoichiometric clusters.

\subsubsection{Direct MEG in Small Clusters}

Using a combination of the Hartree-Fock approximation and the SAC-CI the nature of excited states in different systems can be determined. Realistically sized quantum dots have on the order of 1000 atoms leading to thousands of electronic orbitals in the valence band alone. This poses a substantial computational challenge when taking into account all single and many electron states. In order to surmount this limitation the SAC-CI calculations were only performed on model QDs that consist of about 10 atoms. As a justification for this approximation the single-particle DOS for lead-selenide and cadmium selenide clusters was calculated for systems ranging in size from 8 atoms to 360 atoms. In all cases the different sized clusters exhibited maxima and minima in the DOS at the same energies(JPCC $2008,112,18291)$. Also the optical spectrum of a 10-atom silicon cluster agrees well with the spectra calculated for macroscopic silicon. (PRB 2009, 79, 125332).

In the initial investigation $\mathrm{a} \mathrm{Pb}_{4} \mathrm{Se}_{4}$ and $\mathrm{a} \mathrm{Cd}_{6} \mathrm{Se}_{6}$ cluster were studied. Two things that determine the strength of the electron-electron interactions in semiconducting quantum dots are the quantum confinement and the dielectric screening. Quantum confinement becomes a dominant feature as the size of the quantum dot approaches the size of the exciton Bohr radius, aB. The dielectric screening is given by the dielectric constant, the larger it is the more screening between the charges there is. PbSe has a 
remarkably high Bohr radius, $\mathrm{a}_{\mathrm{B}}=46 \mathrm{~nm}$, and a dielectric constant of $\sim 23$. CdSe has a much lower Bohr radius at $\mathrm{a}_{\mathrm{B}}=5.6 \mathrm{~nm}$ with a dielectric constant of $\sim 6$. While the much larger Bohr radius of $\mathrm{PbSe}$ is indicative of stronger confinement effects, the much larger dielectric constant is suggestive of shielded interparticle interactions and uncorrelated excitons in the photoexcited state.

The results from this investigation are shown in the top two panels of Fig. 3. In the $\mathrm{Pb}_{4} \mathrm{Se}_{4}$ cluster electronic excitations at energies lower that 2.5 times the band gap, Eg, resulted in the production of predominantly single excitons. The threshold for MEG was about 2.6 Eg. This less than ideal threshold suggests that the large dielectric screening is limiting the MEG efficiency. The sharp transition from SEs to MES is further evidence of uncorrelated excitons, as almost all optically excited states between 2.5 and 3 times the band gap become MEs. The results for the $\mathrm{Cd}_{6} \mathrm{Se}_{6}$ cluster, middle panel of Fig. 3, show very different behavior. The MEG onset is higher and exhibits a mixture of single and multiple excitons. This mixture suggests that a significant percentage of CdSe excitations are superpositions of single and multiple excitations and is a result of unscreened many-body interactions due to the low dialectic constant.

Silicon QDs deserve particular attention, since much of the present photovoltaic industry is already based in Si. Experimental measurements on Si QDs put the threshold of MEG at 2.4 times the first band gap excitation energy, and found a quantum yield of 2.6 excitons per photon at 3.4 times $\mathrm{E}_{\mathrm{g}}{ }^{16}$ In order for Si QDs to become a viable option in solar devices, a theoretical understanding of MEG in Si QDs is necessary. Si has a Bohr radius of $4.9 \mathrm{~nm}$ and a dielectric constant of $\sim 12$. First principles calculations show that at 2-3 times the lowest excitation energy the majority of the optically excited states in neutral $\mathrm{Si}_{7}$ take on ME character, bottom panel of Fig. 3, with an MEG threshold of about 2.4 $E_{\mathrm{g}}$. The transition from SEs to MEs is not as sharp PbSe clusters, but it is much more pronounced than in CdSe. Note that the dielectric constant of silicon is between that of lead selenide and cadmium selenide. Many body interactions influence the nature of the phototexcited states more in cadmium selenide QDs and less in lead selenide QDs compared to silicon QDs.

\subsubsection{MEG with Dopants, Defects and Charging}

In order to successfully realize QD photovoltaic cells, detailed analysis of excitations in these materials is needed extending beyond the ideal case. Our calculations establish that photoionization, doping, and surface defects all influence MEG rates, and that these alterations explain the variances observed in experimentally measured MEG rates. In pure ideal semiconductors, excitations are relatively straightforward; an absorbed photon excites an electron across the band gap from the valence band (VB) 
to the conduction band (CB). This produces an interacting electron-hole pair, or exciton, that is stabilized by the Coulomb interaction of the two charges in the semiconducting material. Additionally, if the energy of the incident photon is at least twice the energy of the band gap then MEG can occur.

Deviations from the ideal structure can occur either by design, such as doping $\mathrm{Si}$ with phosphorous or boron to create an n- or p-type material, or by inherent surface defects such as dangling bonds, or through inadvertent photoionization of the material. Materials containing defects exhibit transitions beyond SEs and MEs. Examples of the different types of transitions are presented in the diagram on the left-hand side of Figure 5. If material becomes charged, an extra charge carrier appears in the VB or $\mathrm{CB}$, and additional electronic transitions within these bands become possible. Dopants can introduce a state near the band edge, and surface defects that create dangling bonds can create gap-states, which can be occupied or unoccupied and are not necessarily near the band edge. Each of these examples opens up possibility of intra-band transitions (IB). IB transitions create excitons, but these excitons are contained within either the VB or CB. This renders them useless for photovoltaic applications, which need to separate the charges across the bandgap. They also ensure that at sufficiently high energies excitations are complicated since these IB transitions occur in conjunction with SEs (IB $+\mathrm{SE}$ ). This results in a multi-electron excitation that only creates one new electron-hole pair spanning the bandgap. While formally these transitions are MEs, in photovoltaic applications they are equivalent to SEs.

This section summarizes SAC-CI results found for a negatively charged and n-type doped silicon cluster. The calculations employed the same geometric structure for the neutral and charged clusters, see Figure 4 (inserts). The dopant case is represented by replacing a Si atom from the original cluster with a $\mathrm{P}$ atom. Figure 4 shows the optical spectra generated from the SAC-CI calculations for each of the studied systems. The spectra were obtained non-perturbatively, by explicitly computing the transition dipole moment and oscillator strength for each transition. Generally, the bands seen in spectra are composed of multiple individual excitations. The calculations show that a modification to the cluster from its ideal structure blue-shifts the main absorption peaks in the optical spectra. We have observed blue-shift in the calculated optical spectra with all types of defects including doping, charging, and dangling bonds in variety of systems (JPPC 2011, 115, 10006; CS, 2011, 2, 400; JPCL, 2010,1,232). Other groups have also modeled the same spectral shifts in ionized species (pRB,2000, 62,R16287), as well as reduced photoluminescence in systems with dangling bonds (PNAS, 2009, 106,3011). The effect has been observed experimentally and can be rationalized by Pauli blocking. Here an electron 
occupies the lowest energy CB state in an n-type cluster, preventing a VB electron from making a transition into this state. The effect is similar for positively charged p-type clusters, except it is the lack of a VB electron that prevents the lowest energy exciton transitions. While modifications to the clusters open the possibility for much lower energy IB transitions, these transitions do not appear to have a significant contribution to the absorption spectra.

The contributions of each excited state configuration to the overall character of the excited state in the ideal and modified clusters are presented along the right-hand side of Figure 5. The data points shown are the squares of the expansion coefficients for a given excitation represented in the basis of the single- or multi- electron configurations defined along the left-had side of the same figure. For the ideal $\mathrm{Si}_{7}$ cluster, the transformation from all predominantly SE to ME character occurs at about 2.8 times the band gap energy. In this ideal case the ME contribution is significant across the entire energy range. The $\mathrm{Si}_{7}^{-}$and $\mathrm{Si}_{6} \mathrm{P}$ clusters show almost identical behavior which is to be expected, since doping $\mathrm{Si}$ with $\mathrm{P}$ essentially amounts to adding an extra electron into the CB. Excitation energies in these clusters are smaller than that of the ideal cluster because the lowest excitations in the modified clusters arise due to the IB transitions. As the energy increases, the IB contributions gradually decrease in magnitude, and SEs become the dominant configuration. Most importantly the MEG threshold is pushed beyond $4 \mathrm{E}_{\mathrm{g}}$.

MEG thresholds were also investigated for charged and nonstoichiometric lead selenide cluster s(Jaeger, Chem Sci, JPPC 2011,JPCC2009,split citaitons). It was found that cationic and anionic forms of $\mathrm{Pb}_{4} \mathrm{Se}_{4}$ do not generate multiple excitons at all. The computed MEG threshold for these systems was about $5 \mathrm{E}_{\mathrm{g}}$ which is higher than the ionization energy. This clusters MEG properties were studied when either a lead or selenium atom were removed. These atomic vacancies were found to interfere with MEG. The extent to which the MEG was hindered depended on the bonding properties of the electrons in the vicinity of the lattice defect. In this case, lead's valence electrons carry more angular momentum and are more shielded from the nuclei compared to selenium's valence electrons, assisting in the reformation of bonds. This leads to MEG thresholds with excess lead on the surface being comparable to that of the ideal system. However, when there is excess selenium on the surface MEG is negatively impacted, although the effect is much less severe than that observed in the case of ionization or charging.

\subsection{Impact Ionization Mechanism}

As in bulk materials, MEs in QDs can be created by relaxing a high energy carrier to its ground state and exciting valence electrons across the band gap, and producing additional electron-hole pairs. 
This effect is known as impact ionization (II), and is a main mechanism for MEG and thus the inverse process of MER. The primary difference between exciton multiplication in bulk semiconductors and semiconductor QDs is the conservation of linear (crystal) momentum. In bulk semiconductors not only energy but momentum (k) must be conserved, which increases the ideal energetic threshold of carrier multiplication beyond $2 \mathrm{E}_{\mathrm{g}}$. In QDs, this linear momentum constraint is lifted, reducing the carrier multiplication threshold. The efficiencies of $I$ in semiconductor QDs depend on a couple of different factors. First, the densities of SE and ME states.

\subsubsection{Single and Multiple Exciton Density of States}

We investigated the MEG dynamics caused by the II mechanism in the $\mathrm{Si}_{29} \mathrm{H}_{24}$ QD. The $\mathrm{Si}_{29}$ QD was passivated by hydrogen atoms as shown in the insert of Figure I. The hydrogen surface passivation heals dangling bonds on the bare QD surface, and also provide high-frequency phonon modes due to the light hydrogen atoms. Figure I shows the SE and DE DOS of the $\mathrm{Si}_{29} \mathrm{H}_{24}$ QD. The band gap where the SE DOS starts was calculated as $2.1 \mathrm{eV}$. The lower excitations, up to energies of twice the band gap, are all SEs. The DE DOS starts at twice higher energy than the SE DOS, but increases significantly faster with energy than does the SE DOS due to the increasing combinatorial number of DEs with energy. When initial photoexcitation energy can be exchanged freely between SEs and MEs, most initial population of high energy will flow into DEs, and SEs can appear only at low energies. However, as will be demonstrated below, actual transitions are also influenced by dynamical NA electron-phonon couplings of SE and DE states, and cannot be determined only by the static electronic properties.

\subsubsection{Real-Time Dynamics of Multiple Exciton Generation}

The upper panel of Figure II shows population decrease of total SE population started from an initially excited SE state of the displayed energy. This panel directly exhibits how the MEG appears in the $\mathrm{Si}_{29} \mathrm{H}_{24}$ QD because the lost $\mathrm{SE}$ population all flows into DE states by the II mechanism. Our simulation includes the ground state as well as SEs and DEs, but no population flows into the ground state throughout the current simulation of picoseconds, being in harmony with the experiments. [J. J. Peterson and T. D. Krauss, Nano Lett. 6, 510 (2006); M. Nirmal, B. O. Dabbousi, M. G. Bawendi, J. J. Macklin, J. K. Trautman, T. D. Harris, and L. E. Brus, Nature 383, 802 (1996); M. Sykora, L. Mangolini, R. D. Schaller, U. Kortshagen, D. Jurbergs, and V. I. Klimov, Phys. Rev. Lett. 100, 067401 (2008); I. Moreels, K. Lambert, D. Smeets, D. De Muynck, T. Nollet, J. C. Martins, F. Vanhaecke, A. Vantomme, C. Delerue, G. Allan, et al., ACS Nano 3, 3023 (2009)] The estimated Gaussian and exponential timescales are listed in Table X. 
Table X. Estimated timescales for Gaussian and exponential decay caused by the MEG at different initial excitation energies.

\begin{tabular}{|c|c|c|}
\hline \multirow{2}{*}{ Excitation Energy } & \multicolumn{2}{|c|}{ MEG timescales $(\mathrm{ps})$} \\
\cline { 2 - 3 } & $\tau_{\mathrm{e}}$ & $\tau_{\mathrm{g}}$ \\
\hline $1.8 \mathrm{E}_{\mathrm{g}}$ & 48 & 19 \\
\hline $2.8 \mathrm{E}_{\mathrm{g}}$ & 9.0 & 13 \\
\hline $3.5 \mathrm{E}_{\mathrm{g}}$ & 5.5 & 8.1 \\
\hline
\end{tabular}

The strong energy dependence of the MEG dynamics on the initial excitation energies was obtained by reflecting the rapid increase of the DE DOS with energy. This tendency agrees with the previous experimental and theoretical reports. [A. Franceschetti, J. M. An, and A. Zunger, Nano Lett. 6, 2191 (2006); J. W. Luo, A. Franceschetti, and A. Zunger, Nano Lett. 8, 3174 (2008); E. Rabani and R. Baer, Nano Lett. 8, 4488 (2008); E. Rabani and R. Baer, Chem. Phys. Lett. 496, 227 (2010); C. M. Isborn, S. V. Kilina, X. Li, and O. V. Prezhdo, J. Phys. Chem. C 112, 18291(2008); S. A. Fischer, C. M. Isborn, and O. V. Prezhdo, Chem. Sci. 2, 400 (2011); M. Califano, ACS Nano 3, 2706 (2009);A. Piryatinski and K. A. Velizhanin, J. Chem. Phys. 133, 084508 (2010); M. Califano, ACS Nano 5, 3614 (2011); R. Baer and E. Rabani, Nano Lett. 10, 3277 (2010)] However, exponential population decay by the MEG was assumed in most previous theories which adopt the perturbative method such as Fermi's golden rule. [R. D. Schaller, V. M. Agranovich, and V. I. Klimov, Nature Phys. 1, 189 (2005); A. Franceschetti, J. M. An, and A. Zunger, Nano Lett. 6, 2191 (2006); J. W. Luo, A. Franceschetti, and A. Zunger, Nano Lett. 8, 3174 (2008); E. Rabani and R. Baer, Nano Lett. 8, 4488 (2008); E. Rabani and R. Baer, Chem. Phys. Lett. 496, 227 (2010); A. Piryatinski and K. A. Velizhanin, J. Chem. Phys. 133, 084508 (2010); M. Califano, ACS Nano 5, 3614 (2011); R. Baer and E. Rabani, Nano Lett. 10, 3277 (2010); J. J. H. Pijpers, R. Ulbricht, K. J. Tielrooij, A. Osherov, Y. Golan, C. Delerue, G. Allan, and M. Bonn, Nature Phys. 5, 811 (2009)] The current simulation method which treats the NA couplings nonperturbatively and includes enough number of DEs successfully describes transitions from the initial Gaussian decay to the later exponential decay. The transition appears when enough number of quantum states, SEs and DEs, become involved in the MEG dynamics. It should be remarked that the MEG is caused even with the initial energy lower than the electronic threshold, $2 \mathrm{E}_{\mathrm{g}}$, as seen in the $1.8 \mathrm{E}_{\mathrm{g}}$ case of the upper panel. The real-time phonon dynamics is now explicitly taken into account, allowing this phonon-assisted Auger processes. The lack of the electronic energy, about $0.2 \mathrm{E}_{\mathrm{g}}$, is compensated by the high-frequency Si-H surface modes up to $2000 \mathrm{~cm}^{-1}$ in the $\mathrm{Si}_{29} \mathrm{H}_{24}$ QD. Our result indicates that the 
MEG dynamics is determined not only by the static electronic properties of the QD but also by the dynamical NA phonon couplings. The effect of the surface ligands should become smaller in a larger QD due to the decreased surface-to-volume ratio.

\subsubsection{Real-Time Interplay between the Multiple Exciton Generation and Recombination}

The lower panel of Figure II shows the SE population dynamics as a 2D function of time and energy. The SE population started from the initial excitation of $2.8 \mathrm{E}_{\mathrm{g}}$ exhibits diffusive dynamics to other SEs at the initial stage before $1 \mathrm{ps}$, and then the total SE population decreases reflecting the accelerated MEG drawn in the upper panel. However, the SE population recovers at the lower energy after 2 ps. This population recursion corresponds to the MER, and the recursion energy is identical to 2 $\mathrm{E}_{\mathrm{g}}$, supporting the II mechanism. Since our simulation method simultaneously takes into account both of the MEG and MER, the results involve detailed processes of the combined MEG/MER dynamics, enabling us to study the interplay between the MEG and MER. The MER timescale of a few ps agrees well with extrapolation from the experimental results. [R. D. Schaller, J. M. Pietryga, and V. I. Klimov, Nano Lett. 7, 3469 (2007); I. Robel, R. Gresback, U. Kortshagen, R. D. Schaller, and V. I. Klimov, Phys. Rev. Lett.102, 177404 (2009); Y. Kobayashi, L. Pan, and N. Tamai, J. Phys. Chem. C 113, 11783 (2009); Y. Kobayashi, N. T., Y. H., and N. Tamai, J. Phys. Chem. Lett. 2, 1051 (2011)] If the MER started from a singly excited DE, we would obtained a much longer MER time, hundreds of ps, as we reported.[K. Hyeon-Deuk and O. V. Prezhdo ACS Nano 61239 (2012)] The many DEs produced by the preceding MEG prepare multiple pathways to SEs by couplings to a broader range of SEs, and such MER accelerates significantly. The multiple pathways are particularly essential for the fast and reasonable MER because, in the MER, the density of final SE states is much lower than the density of initial DE states.

\subsection{Phonon-induced Dephasing Mechanism}

\subsubsection{Luminescence, Multiple Exciton Generation, and Multiple Exciton Fission}

The strong Coulombic interactions generate coherent superpositions of excitons in QDs. Such quantum coherence is destroyed by phonon couplings. The phonon-induced dephasing is generally completed in subpicoseconds. Figure 6 shows a diagram showing phonon-induced pure dephasing processes in electronically excited QDs. The left panel illustrates dephasing of the superposition of the ground electronic state and a single exciton (SE) whose dephasing time determines the homogeneous linewidth in luminescence. The middle panel shows superpositions of a high-energy SE and a low-energy ME whose dephasing process contributes to MEG. 
The right panel indicates a superposition of two SEs of similar energy; once the coherence is lost by MEF, the two SE states evolve independently.

The corresponding normalized ACFs (Eq.12), spectral density obtained from the influence spectrum (Eq.13) and cumulant dephasing functions (Eq.14) are shown in Figure 7.35 All the data are calculated at $300 \mathrm{~K}$. A superposition of the ground and excited electronic states created during photoexcitation or luminescence dephases in an ultrafast time, as being reflected by the rapidly decaying ACF and dephasing function in Figure 7. The deduced dephasing time is only about 4 fs. This ultrafast dephasing is mainly attributed to the initial large energy fluctuation, $C_{u}(0)=\left\langle\Delta E^{2}(0)\right\rangle$. The linewidth of the optical signal deduced from this dephasing lifetime is 150 $\mathrm{meV}$ at room temperature, being in good agreement with remarkably broad experimental luminescence linewidth. 44,45 The dephasing MEG process completes even faster, in a few fs. Such ultrafast ME/SE dephasing in QDs could contribute to the MEG process; a ME state can be generated by losing coherence with the initial photoexcited high-energy SE state. The generated ME finally decays into uncorrelated SEs by MEF. The phononinduced dephasing time for MEF is an order of magnitude longer than the above dephasing times for the luminescence and MEG. The calculated timescale is about $54 \mathrm{fs}$. This significantly slower MEF is rationalized by the long-living coherence between the two SE states that have similar energy and orbitals. The small energy difference leads to the initial small energy fluctuation, $C_{u}(0)=\left\langle\Delta E^{2}(0)\right\rangle$ and thus to the slowly decaying dephasing function. After MEF, any excitons no longer have coherence and evolve quite independently. ${ }^{33}$

Fourier transforms of the ACFs provide information about phonon modes involved in dephasing between the coherent two states. The middle panel of Figure 7 indicates that the luminescence and MEG involve both acoustic and optical phonon modes up to high frequencies while MEF needs only low-frequency acoustic modes. Here, the low-dimensionality of the QD allows only approximate classification of acoustic and optical phonon modes. The dephasing related to the luminescence and MEG is mainly contributed by the phonon modes within the 200$600 \mathrm{~cm}^{-1}$ frequency range. These phonon modes correspond not to the hydrogen ligand modes of higher-frequency but to the Si QD core phonons. It can be thus concluded that the main difference among the dephasing rates in the luminescence, MEG and MEF were caused only by the core phonon modes. MEF which dephases two coherent SE states of similar energy and orbitals requires only lower-frequency phonon modes and the spectral density of MEF includes only the lower-frequency core modes up to $200 \mathrm{~cm}^{-1}$.

\subsubsection{Temperature Dependence}

Table I. Estimated dephasing times (fs) at different temperatures.

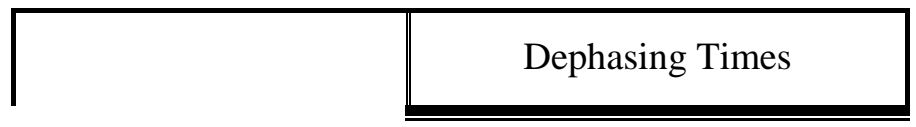




\begin{tabular}{|l|c|c|}
\cline { 2 - 3 } \multicolumn{1}{l|}{} & $300 \mathrm{~K}$ & $80 \mathrm{~K}$ \\
\hline Luminescence $\left(\mathrm{SE} / \mathrm{E}_{\mathrm{g}}\right)$ & $4.00 \pm 0.10$ & $7.00 \pm 0.20$ \\
\hline MEG (ME/SE) & $1.60 \pm 0.05$ & $2.96 \pm 0.09$ \\
\hline MEF (SE/SE) & $54.0 \pm 1.00$ & $205 \pm 0.90$ \\
\hline
\end{tabular}

We show the temperature dependence in the dephasing times of the Si QD in Table I. High temperature activates a broader and high-frequency spectrum of modes, inducing the stronger electron-phonon coupling and thus the faster dephasing. The larger temperature dependence is found in the MEF related to low-frequency acoustic phonon modes than in the luminescence and MEG whose electron-phonon couplings involve broader phonon modes up to the high-frequency optical phonon modes. The MEF exhibits four times faster decoherence at $300 \mathrm{~K}$ than at $80 \mathrm{~K}$, whereas the luminescence and MEG show only twice faster dephasing at the higher temperature. The estimated luminescence linewidth at $80 \mathrm{~K}$ is $80 \mathrm{meV}$ and still agrees with the corresponding experimental data. ${ }^{44,45}$ The difference in the temperature dependence appears because the low-frequency phonons requiring small amounts of thermal energy are more susceptible to the current small temperature change.

\subsubsection{Size Dependence}

The upper panel of Figure 8 shows the Fourier transforms of the ACF to indicate phonon frequencies involved in dephasing of $\mathrm{E}_{\mathrm{g}}$ /ground and $3 \mathrm{Eg} /$ biexciton in the PbSe QD of different size. The PbSe QDs exhibit lower-frequency core modes than the Si QD since $\mathrm{Pb}$ and Se atoms are heavier atoms. Since the low-frequency acoustic modes stem from the QD cores, Figure 8 shows the strong QD size dependency. While the larger PbSe QD includes a wider range of frequencies than the smaller PbSe QD, the intensity of each mode is stronger in the latter than in the former. Thus, the larger electron-phonon coupling is expected in the smaller PbSe QD, supporting the phenomenological elastic model of dephasing in QDs.[Takagahara, T. J. Lumin. 1996, 70, 129]

The corresponding dephasing functions of the larger PbSe QD are plotted in the lower panel of Figure 8. The results were calculated not only using the cumulant approximation but also directly. Both of the results from the cumulant and direct dephasing functions, (14) and (15), coincide well in spite of the long memory in the second-order cumulant (11). The estimated direct dephasing times for $\mathrm{E}_{\mathrm{g}} / \mathrm{ground}$ and $3 \mathrm{Eg}_{\mathrm{g}}$ /biexciton are $9.3 \mathrm{fs}$ and $3.5 \mathrm{fs}$, respectively. The smaller PbSe QD exhibits a bit shorter dephasing times 7.1 fs and 3.2 fs for $E_{\mathrm{g}} /$ ground and $3 \mathrm{E}_{\mathrm{g}}$ /biexciton, respectively, due to its stronger electron-phonon couplings. The dephasing involving biexcitons occurs faster than dephasing involving 
SEs even when the energy difference is similar, about $\mathrm{E}_{\mathrm{g}}$. Biexcitons now consist of higher-energy SEs, and such SEs of high energy are influenced by stronger perturbations caused by lattice phonon modes, leading to the faster dephasing. The homogeneous line width of the luminescence estimated from the dephasing time of $\mathrm{E}_{\mathrm{g}}$ /ground again agree well with the experimental results.[Peterson, J. J.; Krauss, T. D. Nano Lett. 2006, 6, 510]

\subsubsection{Various Electronic States - Bulk, Surface and Plasmon States -}

We consider the phonon-induced dephasing of the three types of electronic states in the $\mathrm{Ag}_{104}$ cluster as the insert of Figure 9 illustrates. The grey distribution indicates the spatial densities of the lowest energy states of each type. The states are classified by their localization with respect to the silver core atoms; the bulk state where the charge density is delocalized over the cluster, the surface state where the charge density is localized on the cluster surface, and the plasmon state where the positive and negative charges are separated at the both sides of the cluster. The characteristic phonon modes coupled to each state can be deduced from the Fourier transforms of the time-dependent fluctuating energy of the corresponding electronic states. The upper panel of Figure 9 shows that the low-frequency acoustic modes mainly contribute to the energy fluctuations of the all three states. The slow acoustic modes largely modulate the whole size and shape of the cluster. Such cluster-scale motions easily affect the electronic states of the above three states whose densities extend through the whole cluster. However, the different strength of the electron-phonon coupling is found depending on the three states. The plasmon state has much smaller amplitude of the spectral density, indicating the weaker electron-phonon couplings. This is rationalized by the charge distribution of the plasmon state which localized away from the cluster core. The largely detached charges can only poorly couple to phonon modes of the silver atoms. In fact, the bulk state whose charge is delocalized over the whole silver cluster and can effectively couple to core phonon modes exhibits the largest spectral intensity.

Table II. Estimated dephasing times (fs) for different electronic states at different temperatures.

\begin{tabular}{|c|c|}
\hline States & Dephasing Times (fs) \\
\hline \hline Bulk, 300 K & 8.7 \\
\hline Surface, 300K & 15.6 \\
\hline Plasmon, 300K & 27.6 \\
\hline Plasmon, 50 K & 82.4 \\
\hline
\end{tabular}


The direct dephasing functions for the lowest-energy bulk, surface, and plasmon states are shown in the lower panel of Figure 9. The bulk state which has the largest phonon intensity decays faster than the other two states, while the plasmon state exhibits the slowest decay reflecting the weakest coupling of phonons to its electronic state. Table II compares their dephasing times at $300 \mathrm{~K}$ and $50 \mathrm{~K}$. The puredephasing times for bulk, surface, and plasmon states at $300 \mathrm{~K}$ directly reflects the differences in the magnitudes of the electron-phonon coupling as indicated in the upper panel. The estimated timescale of the plasmon state is in harmony with the experimental data. [M. Z. Liu, M. Pelton, and P. GuyotSionnest, Phys. Rev. B 79,035418 (2009)] The temperature affects the phonon dynamics of the $\mathrm{Ag}_{104}$, and thus the dephasing time scales. The plasmon pure-dephasing times for $\mathrm{Ag}_{104}$ differs by almost three times at $300 \mathrm{~K}$ and $50 \mathrm{~K}$. 


\section{Electron Phonon Relaxation}

Due to the broad bandwidth of the solar spectrum, electrons in semiconductor photovoltaic materials can be excited into different energy levels in the conduction band. These excited electrons typically lose excess energy rapidly to the lattice via a series of electron-phonon scattering events. To reduce this thermalization loss for possible solar efficiency enhancement, the electron relaxation rate should be minimized. ${ }^{3,8,25}$ The intrinsic electron-phonon coupling for a bulk material cannot be modified. However, making the semiconductors into nanostructures such as QDs opens up the possibility of decoupling electrons and phonons. Due to quantum confinement effects, the electronic structure of QDs exhibits discrete energy levels, and the spacing between adjacent levels may become larger than the energy of a single phonon. In this case only multiphonon processes will be able to relax the electrons, potentially slowing down the relaxation rate compared to bulk materials. 3, 26, 27

Experiments have also shown that both electron-phonon and Auger-type processes can coexist in the electron relaxation process in nanocrystals. ${ }^{28}$ Depending on types of the materials, status of surface passivation, and types of surface ligands, either the electron-phonon process or the Auger process can dominate the charge carrier relaxation. However, the Auger process can be avoided if electrons and holes are separated, or if electrons and holes have a similar DOS. Current experimental methods can enable effective separation of electrons and holes, making it possible to decouple the Auger and electron-phonon relaxation processes in nanocrystals. ${ }^{28,} 29$ Slowing down electron-phonon coupling is favored for MEG efficiency and enhanced solar cell efficiency in general.

Due to the fundamental and practical importance of electron-phonon coupling in photovoltaic nanomaterials, it is crucial to understand how the electron-phonon relaxation depends on a variety of factors including material, temperature, nanoparticle size and shape, surface terminations, surfactants, etc. ${ }^{9}$ Recently, a nonadiabatic molecular dynamics approach has been developed ${ }^{11,30}$ to simulate the electron relaxation process in QDs, ${ }^{31-33}$ and this process has been used to investigate several different systems and is discussed below. For further reading consult references. ${ }^{31-40}$

In all of the simulations discussed in this section the TDDFT-FSSH theory was implemented with the VASP ${ }^{41}$ DFT package. The simulations were performed with either Perdew and Wang ${ }^{42}$ or PBE exchange-correlation functionals, Vanderbilt pseudopotentials, ${ }^{43}$ and a converged plane-wave basis. The simulations were carried out in a cubic cell periodically replicated in three dimensions, as stipulated by the plane wave basis. To prevent spurious interactions between periodic images of the QD, the cell was constructed to have at least $8 \dot{A}$ of vacuum between the QD relplicas. 
The QDs are initially constructed at zero temperature from the bulk structures and bond lengths, and then relaxed to their lowest energy configuration. This relaxed configuration is then heated to a temperature, usually $300 \mathrm{~K}$, with repeated velocity rescaling. After the system is heated a groundelectronic state microcanonical trajectory is performed for several picoseconds. The transition dipole moments and oscillator strengths for excitations between the KS orbitals were computed and used both to generate the optical absorption spectrum, and to pick the most optically active excitations for the initial conditions of the NA runs. A 1 fs nuclear and a $10^{-3}$ fs electronic time step was used for the dynamics calculations. The data shown in the figures below are converged by averaging over at least 500 runs. Further details can be found in many references. ${ }^{11,31-40,44,45}$

\subsection{Phonon-Assisted Relaxation of Charge Carriers in PbSe}

Of all semiconducting nanomaterials studied lead salts, such as $\mathrm{PbS}$ and $\mathrm{PbSe}$, show some of the most unique electronic and transport properties. ${ }^{46-50}$ Their conduction and valence bands are more symmetric than other semiconductor materials, such as CdSe, potentially rendering Auger relaxation processes inefficient. ${ }^{51}$ Also the effective mass of electrons and holes in lead salts are similar and small, 52 promising strong quantum confinement effects and inducing quantization of bulk electronic bands. ${ }^{48}$ This discretization was expected to produce a mismatch between the electronic and vibrational energy quanta and is expected to produce a phonon bottleneck in the charge-carrier relaxation. ${ }^{3}$ Both of these attributes favor MEG, and indeed high quantum yields in this material were observed. ${ }^{5,6,19-21}$ However, ultrafast intraband charge-phonon relaxation in PbSe QDs was observed suggesting that no phonon bottleneck exsisted. ${ }^{53,54}$ Taking a step further beyond static EMT and pseudopotential calculations, timedomain theoretical studies where able to directly mimic time-resolved experiments and provide detailed atomistic description of the relaxation process.

Phonon motions induce fluctuations in electronic DOS, as illustrated with $\mathrm{Pb}_{68} \mathrm{Se}_{68}$ in the top panel of Figure 10. The fluctuations are minor: $\mathrm{CB}$ and VB edges change by less than $0.1 \mathrm{eV}$ and are significantly smoothed (Figure 10). Phonon motions mix states of different symmetries and reduce gaps between states. The bottom panel of Figure 10 presents NA dynamics of electron and hole relaxation mediated by coupling to phonons. ${ }^{8,31,33}$ Charge carriers visit multiple states during relaxation, and no intermediate states play special roles. The population peaks created by photoexcitation spread to reappear near the band-gap. Relaxation is nearly complete within a picosecond, in agreement with experiment. $^{53,55}$ No phonon bottleneck is observed at high photoexcitation energies. Most likely transitions involve small amounts of energy that are close to a phonon energy of $100-200 \mathrm{~cm}^{-1}(12-25$ 
$\mathrm{meV}$ ). This indicates that electronic energy gaps are small at high energies, explaining the absence of the phonon bottleneck. Occasionally, up to $0.3-0.6 \mathrm{eV}$ of electronic energy can be lost to phonons in single events. ${ }^{8,31,33}$ Multiphonon relaxation was initially proposed to rationalize ultrafast experimental data, ${ }^{53}$ and is indeed seen in our simulation; however, faster resonant electron-phonon energy exchange is also efficient, particularly at higher energies.

\subsection{Plasmon Relaxation}

Beyond semiconductors, noble metal nanoparticles have been studied for a wide variety of applications. These systems exhibit collective excitations known as plasmons, and are responsible for many of the novel electronic and optical properties exhibited by noble metal nanoparticles. Some of the applications that have received attention are data storage, lasing, electro-optics, biological imaging, and light harvesting for solar cells. Just as in their semiconductor counterparts electron-phonon dynamics play a lead role in many of their primary applications. The electron-phonon relaxation rate is a central parameter in many devices. It controls the response time in electro-optic switches. Electron-phonon coupling causes nonradiative relaxation and heating in electronic, optical, and photovoltaic devices. In these systems this is an undesirable attribute and the goal is to minimize this effect. However in photothermal therapy metal nanoparticles ability to rapidly convert an absorbed photon into energy and heat is a desirable feature to capitalize on. Therefore, it is necessary to learn the mechanisms behind plasmon-phonon relaxation in metal nanoclusters in order to ascertain if there is possibility of tuning properties for specific applications.

The Fourier transforms of the plasmon state energies, and the results are shown in Figure 11. The vibrational modes that modulate the energy levels create the largest couplings. It was found that the plasmon excitations in the $\mathrm{Ag}_{68}$ nanoparticles couple exclusively to low-frequency phonon modes. It is these low-frequency modes that cause plasmons to relax in energy. In all cases the dominate modes have frequencies less than $100 \mathrm{~cm}^{-1}$. This is in contrast to PbSe and $\mathrm{Si}$ nanocrystals where in addition to acoustic phonons, optical modes around $200 \mathrm{~cm}^{-1}$ also have strong influence in charge carrier relaxation. The dominance of slow acoustic modes in the plasmon relaxation process is rationalized because the low frequency vibrations modulate the size and shape of the nanoclusters. High-frequency optical modes involve local displacements of atoms relative to each other, and have little influence over the global nanoclusters properties. Since plasmon states are delocalized across the entire dot they couple most strongly to the slow acoustic modes. The variations in the symmetry of the different plasmon states explains the differences seen the frequencies contributing to electron-phonon relaxation. The overall 
trend observed is that lower energy plasmon states tend to couple more strongly to phonons compared to the higher energy states. The higher energy states are more delocalized from the nanoparticle, and are less affected by atomic motions taking place inside the particle.

The relaxation times of both population and energy are shown in Table X. The plasmon population decay is obtained by summing TD populations of all plasmon states, and the decay results from population transfer into nonplasmon states. We see that the lower energy plasmon states relax out of the plasmon band more quickly than the high energy states. The higher energy plasmon excitations take time to traverse the manifold of plasmon states before decaying into the low energy bulk states The states that undergo the quickest population relaxation are also the states that coupled most strongly to the phonon. The energy relaxation exhibits little dependence on the initial conditions. In all cases relaxation took place on the ps time scale similar to semiconductor QDs.

\subsection{Temperature Dependence}

Temperature dependence of carrier relaxation distinguishes phonon-induced processes from other channels, such as Auger scattering. ${ }^{56}$ Experiments have shown ${ }^{29,53}$ that relaxation is much more temperature-dependent in $\mathrm{PbSe}$ than in $\mathrm{CdSe} \mathrm{QDs}$, where Auger processes are more effective. Different temperature dependencies suggest that phonons play an important role in most, but not all, QDs. Temperature dependence of electron-phonon relaxation was looked at in $\mathrm{Pb}_{16} \mathrm{Se}_{16}{ }^{35}$ and $\mathrm{Cd}_{33} \mathrm{Se}_{33}{ }^{36}$ QDs. Several things were ascertained from these studies, many ubiquitous for both systems.

The phonon modes that induce charge carrier relaxation are obtained by taking the Fourier transform of the initially populated energy level. The electron phonon coupling, $\boldsymbol{d}_{\boldsymbol{k} m}$ in Eqs. 7 and 8, is directly related to the second derivative of the energy along the nuclear trajectory, and therefore those vibrational modes that most strongly modulate the energy levels create the largest coupling. ${ }^{57}$ Figure 12 shows the Fourier transform of the LUMO vibrations for the CdSe QD. ${ }^{36}$ All of the conclusions drawn from Figure 12 hold for the spectral density of PbSe as well. Temperature affects the coupling in two ways. First, at higher temperature, the Fourier transforms show a high-frequency tail, indicating that a larger fraction of high-frequency surface modes are involved in the carrier decay dynamics. This can be understood by considering that increased temperature can activate higher-frequency modes. Second, the Fourier transform curves at higher temperature are broadened, indicating that more modes are modulating the energy levels. Both the increase in the number of participating modes and the increased coupling to high-frequency modes would suggest an increase in relaxation rate with temperature. This is indeed what was found for both CdSe and PbSe, and is shown if Figure 13 for PbSe. 
These studies allowed for the development of a quantitative model on the temperature dependence of hot-carrier decay in nanocrystals. ${ }^{35,36}$ Notice that from Eq. 8, if we assume $\boldsymbol{d}_{\boldsymbol{k} m}$ is only implicitly dependent on temperature, the NA coupling strength is proportional to the ion velocities $\dot{\boldsymbol{R}}$, and in turn to the square root of kinetic energy $E_{k}$. Since the MD process treats the ions classically, MD temperature $T_{M D}$ is proportional to $E_{K}$. From the time-dependent perturbation theory, a simple estimation is that the transition probability is proportional to the square of the off-diagonal element of the perturbation matrix, which is $|N A|^{2}$ in our case. Based on the above considerations the temperature dependence of the hot carrier relaxation rate could be simply written as

$$
\gamma \sim|N A|^{2} \sim\left|\boldsymbol{d}_{\boldsymbol{k} m}\right|^{2}|\dot{\boldsymbol{R}}|^{2} \sim\left|\boldsymbol{d}_{\boldsymbol{k m}}\right|^{2} T_{M D}
$$

where, $\gamma$, NA, $\boldsymbol{d}_{\boldsymbol{k}}, \dot{\boldsymbol{R}}$, and $T_{M D}$ represent the relaxation rate, nonadiabatic coupling, electron-phonon coupling, ion velocity and temperature, respectively.

However with both $\mathrm{PbSe}$ and $\mathrm{CdSe}$ calculated results deviated significantly from this expected trend. For both systems relaxation rates were better fitted to $T_{M D}^{0.4}$ than to $T_{M D}$ (Figure $14 \mathrm{PbSe}$ ). The weaker temperature dependence can only be attributed to the temperature dependence of electronphonon coupling, $\boldsymbol{d}_{\boldsymbol{k}}$, strength. To investigate this term, we calculated the NA coupling strength between pairs of states for each system, and found that it decrease with temperature as $\mathrm{T}^{-0.3}$. Although QD geometry is weakly temperature-dependent, the coupling can strongly depend on QD expansion. Figure 14 also shows that the hole relax more quickly than the electrons. This is explained by denser hole states.

Experimental results show no temperature dependence for CdSe QDs, and a much weaker temperature dependence at low temperatures for $\mathrm{PbSe} .{ }^{53} \mathrm{In} \mathrm{PbSe}$ QDs, at low temperatures the Auger channel is more efficient than the multiphonon channel, which gives the weak temperature dependence. At higher temperature, when the electron-phonon channel becomes more efficient, it contributes to the overall relaxation rate and stronger temperature dependence is observed. This confirms that CdSe QDs relax predominantly from Auger relaxation channels. (*Better Transition*)

\subsection{Phonon Bottleneck and the Quantum Zeno Effect}

However current experimental methods can enable effective separation of electrons and holes, making it possible to decouple the Auger and electron-phonon relaxation processes in NCs. For CdSe nanocrystals, with a surfactant of a deep hole trapping molecule, such as pyridine, the electron-phonon 
interaction is dominant, and a slow relaxation of $\sim 200 \mathrm{ps}$ is observed. ${ }^{28,29}$ This is orders of magnitude longer than that in the bulk system. Panday and Guyot-Sionnest have found that coating CdSe QDs with thick shells of multiple $\mathrm{ZnS}$ and $\mathrm{ZnSe}$ layers protected the surface of the QD core from the environment. This serves to suppress the ligand mediated relaxation channel. The authors also suggested that the shells trapped the holes, decoupling them from the core-bound electrons and suppressing Auger relaxation. Upon performing a DOS analysis on both a CdSe QD and a CdSe/ZnS core/shell QD the ZnS shell creates additional VB states near the edge of the band gap. The electronic structure near the CB edge remains unaffected, supporting the electron-hole separation concept (Figure 15).

When the semiclassical decoherence correction is included in TDDFT-NAMD a phonon bottleneck between the $1 \mathrm{P}_{\mathrm{e}}$ and $1 \mathrm{~S}_{\mathrm{e}}$ in a CdSe QD was observed in a FSSH study. Using the optical response formalism the pure-dephasing time between these states was as $36 \mathrm{fs}$. When this dephasing time was incorporated into the NAMD, the lifetime of the 1Pe state was calculated to be 670ps, in good agreement with the measured $1 \mathrm{~ns}$ lifetime. It was also found that if the dephasing time were increased the relaxation time sped up, Figure 16. Dephasing can be understood as a measurement of the state of the electronic subsystem performed by the phonon environment. A fast decoherence then corresponds to frequent measurements of the quantum-mechanical state. As demonstrated a fast decoherence also leads to reduced relaxation rates. This principle where a system in a known initial state can have its evolution "frozen" by frequent measurements is known as the quantum Zeno effect. It is the suppression of unitary time evolution caused by quantum decoherence within the system (PRA,65,013404). The quantum Zeno effect rationalized the phonon bottleneck seen in semiconductor QDs (Kilina Paper).

\subsection{Core/Shell to control excited state dynamics}

Another example of controlling the optical properties of a system by tuning is surface composition was demonstrated by Wei et al. It was found that band edge emission was quenched in sulfur terminated CdS QDs and fully recovered when QDs were cadmium terminated. In both cases the absorption spectra remained largely unaffected. The hypothesis was that the termination of the CdS QDs with a sulfur shell introduces surface trap states that provide for effective nonradiative recombination pathways. To test this conjecture, density of states (DOS) calculations for $\mathrm{Cd}$ and $\mathrm{S}$ energy states were implemented on representative CdS QDs. The dots had either a stoichiometric surface, or were terminated with predominately $\mathrm{Cd}$ or $\mathrm{S}$. The $\mathrm{Cd}$ and $\mathrm{S}$-rich clusters were simulated by using the coordinates for the ideal QD and replacing one, three or five $\mathrm{Cd}(\mathrm{S})$ atoms with $\mathrm{S}(\mathrm{Cd})$ atoms. These modified structures were then each relaxed to their lowest energy state. 
When the larger Cd atoms were used to replace the smaller S atoms the size of the QD increased slightly. The energy gap narrowed due to both decreased quantum confinement and the appearance of defect states near the edges of the valence and conduction bands. Despite the band gap narrowing, a well-defined energy gap remained, with no evidence of a midgap surface trap states. On the other hand,as the surface was made increasingly S-rich, numerous midgap surface states began to appear in the DOS, Figure 17. Time-domain ab initio simulations demonstrate that these midgap states should provide an efficient nonradiative recombination pathway for electrons and holes. Thus it was concluded that in the S-rich QDs, efficient nonradiative recombination was mediated by the midgap surface effectively quenching fluorescence. TDDFT calculations were performed to calculate the absorption spectra and it was found that the midgap states were largely optically inactive, explaining the very little change in the absorption spectra observed experimentally.

\subsection{Ligands Saturate dangling Bonds and Accelerate Electron-phonon Relaxation}

Ligand contribution to QD excitation dynamics is exemplified with Si and Ge QDs. Ab initio calculations showed ${ }^{32}$ that quantum confinement makes the electron and hole DOS more symmetric in Si and Ge QDs compared with bulk. Despite symmetric DOS, electrons decay faster than holes shown in Figure 18 for Si. Asymmetric relaxation can be rationalized by stronger electron-phonon coupling in the $\mathrm{CB}$, due to larger contributions of high-frequency phonons associated with $\mathrm{Ge}-\mathrm{H}$ and $\mathrm{Si}-\mathrm{H}$ surface bonds. As can be seen in the inserts in the right panel of Figure 16 the CB states for Si are more delocalized on the surface than the VB states. The rest of Figure 16 shows the spectral densities of the phonon modes that couple to $\mathrm{CB}$ and VB states of $\mathrm{Sie}_{29} \mathrm{H}_{24}$. Low-frequency motions are $\mathrm{Si}$-Si bond vibrations and show little difference between VB and CB, left panels. However, asymmetry is clearly seen in high-frequency components that originate from Si-H surface bonds (right panels of Figure 19). The electrons in the conduction band are going to couple much more strongly to these high-frequency modes as compared to their counterpart holes in the VB. The higher frequency modes have higher velocities and accelerate charge relaxation through the velocity dependence of NA coupling, allowing electrons to relax faster than holes. ${ }^{11}$

A more recent study of CdSe also confirms that ligands tend to increase relaxation rates. ${ }^{38}$ Photoexcited dynamics up to $1 \mathrm{ps}$ were studied in a $\mathrm{Cd}_{33} \mathrm{Se}_{33}$ cluster passivated by commonly used ligands, $\left(\mathrm{OPMe}_{3}\right)$ and primary amines $\left(\mathrm{NH}_{2} \mathrm{Me}\right)$, and compared to the ligand free system. It was found that the ligands introduce a manifold of high energy hybridized electronic states. Figure 17 shows that relaxation occurs noticeably faster in the litigated QDs. This fast relaxation in the passivated dots originates from the 
strong electron-phonon couplings provided by the hybridized states. These hybridized states couple to both high-frequency vibrations associated with the ligands and low frequency phonon modes in the cluster. Even if an electron or hole is is not initially excited into a hybridized orbital, the NA couplings between the electronic states forces the charge to jump to one of the neighboring hybridized orbitals, thus opening new relaxation channels. These hybridized states start closer to the band gap in the $\mathrm{NH}_{2} \mathrm{Me}$ passivated dot compared to the $\mathrm{OpMe}_{3}$ passivated, allowing the amine litigated system to relax faster at $2.5 \mathrm{E}_{\mathrm{g}}$, Figure 20 . At $3 \mathrm{E}_{\mathrm{g}}$ the hybridized states in both valence and conduction bands contributed equally to electron-phonon couplings in both litigated systems; both relaxation rates become nearly identical between the two systems at this excitation energy.

\section{Conclusion}

Valuable insights into semiconductor QD properties can be ascertained from a $b$ initio modeling of excited-state dynamics in the energy and time domains. Photoexcited processes dominate the excitation and charge dynamics in nanoscale systems. The atomistic methods reviewed in this paper are ideal for modeling defects, ligands, charges, dopants, and dangling bonds in QDs leading. This leads to an enhanced understanding of the properties of nanoscale devices creating valuable insights for development of a multitude of applications.

Electron-electron Coulombic interactions open up the possibility MEG upon the absorption of a single photon. Using SAC-CI, this Coulomb correlation is able to be directly accounted for. The nature of the excited states of different materials such as $\mathrm{CdSe}, \mathrm{PbSe}$, and $\mathrm{Si}$ depend on both quantum confinement and dielectric shielding. It was found that QD charging gives rise to intraband transitions that exhibit little optical activity but dramatically modify the nature and energy of the excited electronic states. Charging also blue-shifts absorption spectra and increases ME thresholds. Surface reconstructions also significantly changes QD bandgaps.

Dephasing and relaxation are the two distinct processes that are induced by electron-phonon interactions. Superpositions of electronic states, created by the Coulomb interaction during photoexcitation and the subsequent time evolution, dephase into incoherent mixtures of states on a time scale of 10's of fs, Figure 20. Dephasing is ultrafast if it involves electronic states with substantially different energies and spatial densities. Examples of this include superpositions of SEs and MEs and ground and excited states. ME fission into independent SEs occurs by dephasing that is much slower. This is because MEs are typically formed by SEs that are close in energy. 
Photodriven exciton multiplication and subsequent phonon-induced pure-dephasing occur very rapidly in semiconductor QDs. This is followed by competition between impact ionization and exciton thermalization which allow the system to reach the band edge within 10 ps in most systems. Our studies indicate that Auger processes are an essential component to MEG. Until very recently, they had only been modeled in the time-independent approach. The newly developed sparse matrix techniques discussed in this chapter allow the study of time-domain simulations of Auger processes that inherently involve many electronic states. These studies have helped to validate standard rate theory models that only incorporate exponential decay. The electron-phonon relaxation pathway is dominant until lower energies. Since the coupling between SEs and MEs promotes both forward and backward processes, the directionality is highly influenced by the relative DOS for SEs and MEs. At high energies, SEs generate MEs, while at low energies MEs annihilate to form SEs.

TDDFT combined with NAMD simulates the complex evolutions of coupled electronic and vibrational degrees of freedom as it occurs in nature. Time domain modeling of electron-phonon relaxation unified two seemingly contradicting experimental observations: despite large spacing between optical lines, a phonon bottleneck of electron-phonon relaxation exists only under very special conditions. QD spectra are composed of multiple individual excitations that combine into distinct bands according to optical selection rules. Selection rules are much less stringent for electron-phønøn transitions. Even though relatively few excitations are strongly optically active, most excited electronic states participate in phonon relaxation. At the band edges, the decoherence discussed about above has to be included in relaxation dynamics. When this happens, a bottleneck is found, and can be experimentally found when a shell is added to the QD to separate electron and hole states. Through these studies, it was found that ligand and shell layers saturate surface dangling bonds and alter high-energy regions of CB and VB. While shells can often isolate electrons and holes from high-frequency ligand modes, and ligands can create strong electron-phonon coupling which speeds up electron-phonon relaxation. Studies like the ones in this review elucidate processes dominant in specific systems allowing for rational design of new applications

With increased computational power, the current methods will be applied to larger QDs and more diverse materials involving realistic ligands, surface defects and viable core/shell compositions. Longer-scale simulations of luminescence quenching and the phonon bottleneck will become possible. Photovoltaic assemblies of QDs with other materials, such as molecular chromophores, polymers, and inorganic semiconductors present new theoretical questions. The issues raised here with electronic states 
in semiconductor QDs find close similarities with spin states of metallic QDs. Ab initio approaches in time and energy domains will greatly advance our understanding of QD properties that govern solar energy harvesting, photovoltaics, lasing, spintronics, and bio-imaging.

\section{Acknowledgements}

The research was supported by the National Science Foundation, US Department of Energy and the KAKENHI No. 24750016. We thank colleagues and collaborators: Sean A. Fischer, Angeline B. Madrid, Christine M. Isborn, Svetlana V. Kilina, Xiaosong Li, Hideyuki Kamisaka, Koichi Yamashita, Kim Hyeon-Deuk, and Bradley F. Habenicht, Hua Bao, Xiulin Ruan, Dimitri S. Kilin, Colleen F. Craig, Liangliang Chen, and Taizhi Tan for the contributions.

\section{References}

1. D. J. Milliron, S. M. Hughes, Y. Cui, L. Manna, J. Li, L.-W. Wang and A. Paul Alivisatos, Nature, 2004, 430, 190-195.

2. D. S. Kilin, O. V. Prezhdo and Y. Xia, Chemical Physics Letters, 2008, 458, 113-116.

3. A. J. Nozik, Annual review of physical chemistry, 2001, 52, 193-231.

4. A. Pandey and P. Guyot-Sionnest, Science, 2008, 322, 929-932.

5. $\quad$ R. D. Schaller and V. I. Klimov, Physical Review Letters, 2004, 92, 186601.

6. R. J. Ellingson, M. C. Beard, J. C. Johnson, P. Yu, O. I. Micic, A. J. Nozik, A. Shabaev and A. L. Efros, Nano Letters, 2005, 5, 865-871.

7. J. A. McGuire, J. Joo, J. M. Pietryga, R. D. Schaller and V. I. Klimov, Accounts of Chemical Research, 2008, 41, 1810-1819.

8. $\quad$ O. V. Prezdho, Chemical Physics Letters, 2008, 460, 1-9.

9. O. V. Prezhdo, Accounts of Chemical Research, 2009, 42, 2005-2016.

10. K. Hyeon-Deuk, Oleg, Journal of Physics: Condensed Matter, 2012, 24.

11. C. F. Craig, W. R. Duncan and O. V. Prezhdo, Physical Review Letters, 2005, 95, 163001.

12. M. A. L. Marques and E. K. U. Gross, Time-dependent density functional theory, Annual Reviews, Palo Alto, CA, 2004.

13. S. Hammes-Schiffer and J. C. Tully, The Journal of Chemical Physics, 1994, 101, 4657-4667.

14. J. C. Tully, The Journal of Chemical Physics, 1990, 93, 1061-1071.

15. P. V. Parahdekar and J. C. Tully, J. Chem. Phys, 2005, 122.

16. B.-R. Hyun, Y.-W. Zhong, A. C. Bartnik, L. Sun, H. D. Abruña, F. W. Wise, J. D. Goodreau, J. R. Matthews, T. M. Leslie and N. F. Borrelli, ACS Nano, 2008, 2, 2206-2212.

17. G. I. Koleilat, L. Levina, H. Shukla, S. H. Myrskog, S. Hinds, A. G. Pattantyus-Abraham and E. H. Sargent, ACS Nano, 2008, 2, 833-840.

18. E. Rabani and R. Baer, Nano Letters, 2008, 8, 4488-4492.

19. R. D. Schaller, M. Sykora, J. M. Pietryga and V. I. Klimov, Nano Letters, 2006, 6, 424-429.

20. R. D. Schaller, V. M. Agranovich and V. I. Klimov, Nat Phys, 2005, 1, 189-194.

21. J. E. Murphy, M. C. Beard, A. G. Norman, S. P. Ahrenkiel, J. C. Johnson, P. Yu, O. I. Mićić, R. J. Ellingson and A. J. Nozik, Journal of the American Chemical Society, 2006, 128, 3241-3247.

22. C. M. Isborn, S. V. Kilina, X. Li and O. V. Prezhdo, The Journal of Physical Chemistry C, 2008, 112, 1829118294.

23. J. M. An, M. Califano, A. Franceschetti and A. Zunger, The Journal of Chemical Physics, 2008, 128, 164720-164727.

24. A. Franceschetti, J. M. An and A. Zunger, Nano Letters, 2006, 6, 2191-2195.

25. J. Nelson, The Physics of Solar Cells, Imperial College Press, London, 2003. 
26. J. Urayama, T. B. Norris, J. Singh and P. Bhattacharya, Physical Review Letters, 2001, 86, 4930-4933.

27. A. D. Yoffe, Advances in Physics, 2001, 50, 1-208.

28. P. Guyot-Sionnest, M. Shim, C. Matranga and M. Hines, Physical Review B, 1999, 60, R2181-R2184.

29. P. Guyot-Sionnest, B. Wehrenberg and D. Yu, The Journal of Chemical Physics, 2005, 123, 074709074707.

30. S. A. Fischer, B. F. Habenicht, A. B. Madrid, W. R. Duncan and O. V. Prezhdo, The Journal of Chemical Physics, 2011, 134, 024102-024109.

31. S. V. Kilina, C. F. Craig, D. S. Kilin and O. V. Prezhdo, The Journal of Physical Chemistry C, 2007, 111, 48714878.

32. K. Hyeon-Deuk, A. B. Madrid and O. V. Prezhdo, Dalton Transactions, 2009, 10069-10077.

33. S. V. Kilina, D. S. Kilin and O. V. Prezhdo, ACS Nano, 2008, 3, 93-99.

34. B. F. Habenicht and O. V. Prezhdo, Physical Review Letters, 2008, 100, 197402.

35. H. Bao, B. F. Habenicht, O. V. Prezhdo and X. Ruan, Physical Review B, 2009, 79, 235306.

36. L. Chen, H. Bao, T. Tan, O. V. Prezhdo and X. Ruan, The Journal of Physical Chemistry C, 2011, 115, 11400-11406.

37. A. J. Neukirch, Z. Guo and O. V. Prezhdo, The Journal of Physical Chemistry C, 2012, 116, 15034-15040.

38. S. Kilina, K. A. Velizhanin, S. Ivanov, O. V. Prezhdo and S. Tretiak, ACS Nano, 2012, 6, 6515-6524.

39. R. Long and O. V. Prezhdo, Journal of the American Chemical Society, 2011, 133, 19240-19249.

40. S. V. Kilina, D. S. Kilin, V. V. Prezhdo and O. V. Prezhdo, The Journal of Physical Chemistry C, 2011, 115, 21641-21651.

41. G. Kresse and J. Furthmüller, Physical Review B, 1996, 54, 11169-11186.

42. J. P. Perdew, J. A. Chevary, S. H. Vosko, K. A. Jackson, M. R. Pederson, D. J. Singh and C. Fiolhais, Physical Review $B, 1992,46,6671-6687$.

43. D. Vanderbilt, Physical Review B, 1990, 41, 7892-7895.

44. B. F. Habenicht, C. F. Craig and O. V. Prezhdo, Physical Review Letters, 2006, 96, 187401.

45. W. R. Duncan, C. F. Craig and O. V. Prezhdo, Journal of the American Chemical Society, 2007, 129, 85288543.

46. J. J. Peterson and T. D. Krauss, Physical Chemistry Chemical Physics, 2006, 8, 3851-3856.

47. W. Lu, J. Fang, Y. Ding and Z. L. Wang, The Journal of Physical Chemistry B, 2005, 109, 19219-19222.

48. F. W. Wise, Accounts of Chemical Research, 2000, 33, 773-780.

49. B. L. Wehrenberg, C. Wang and P. Guyot-Sionnest, The Journal of Physical Chemistry B, 2002, 106, 10634-10640.

50. H. Zeng, Z. A. Schelly, K. Ueno-Noto and D. S. Marynick, The Journal of Physical Chemistry A, 2005, 109, 1616-1620.

51. V. I. Klimov, A. A. Mikhailovsky, D. W. McBranch, C. A. Leatherdale and M. G. Bawendi, Science, 2000, 287, 1011-1013.

52. P. Liljeroth, P. A. Z. van Emmichoven, S. G. Hickey, H. Weller, B. Grandidier, G. Allan and D. Vanmaekelbergh, Physical Review Letters, 2005, 95, 086801.

53. R. D. Schaller, J. M. Pietryga, S. V. Goupalov, M. A. Petruska, S. A. Ivanov and V. I. Klimov, Physical Review Letters, 2005, 95, 196401.

54. J. M. Harbold, H. Du, T. D. Krauss, K.-S. Cho, C. B. Murray and F. W. Wise, Physical Review B, 2005, 72, 195312.

55. R. R. Cooney, S. L. Sewall, K. E. H. Anderson, E. A. Dias and P. Kambhampati, Physical Review Letters, 2007, 98, 177403.

56. A. L. Efros, V. A. Kharchenko and M. Rosen, Solid State Communications, 1995, 93, 281-284.

57. W. H. Miller and T. F. George, The Journal of Chemical Physics, 1972, 56, 5637-5652. 


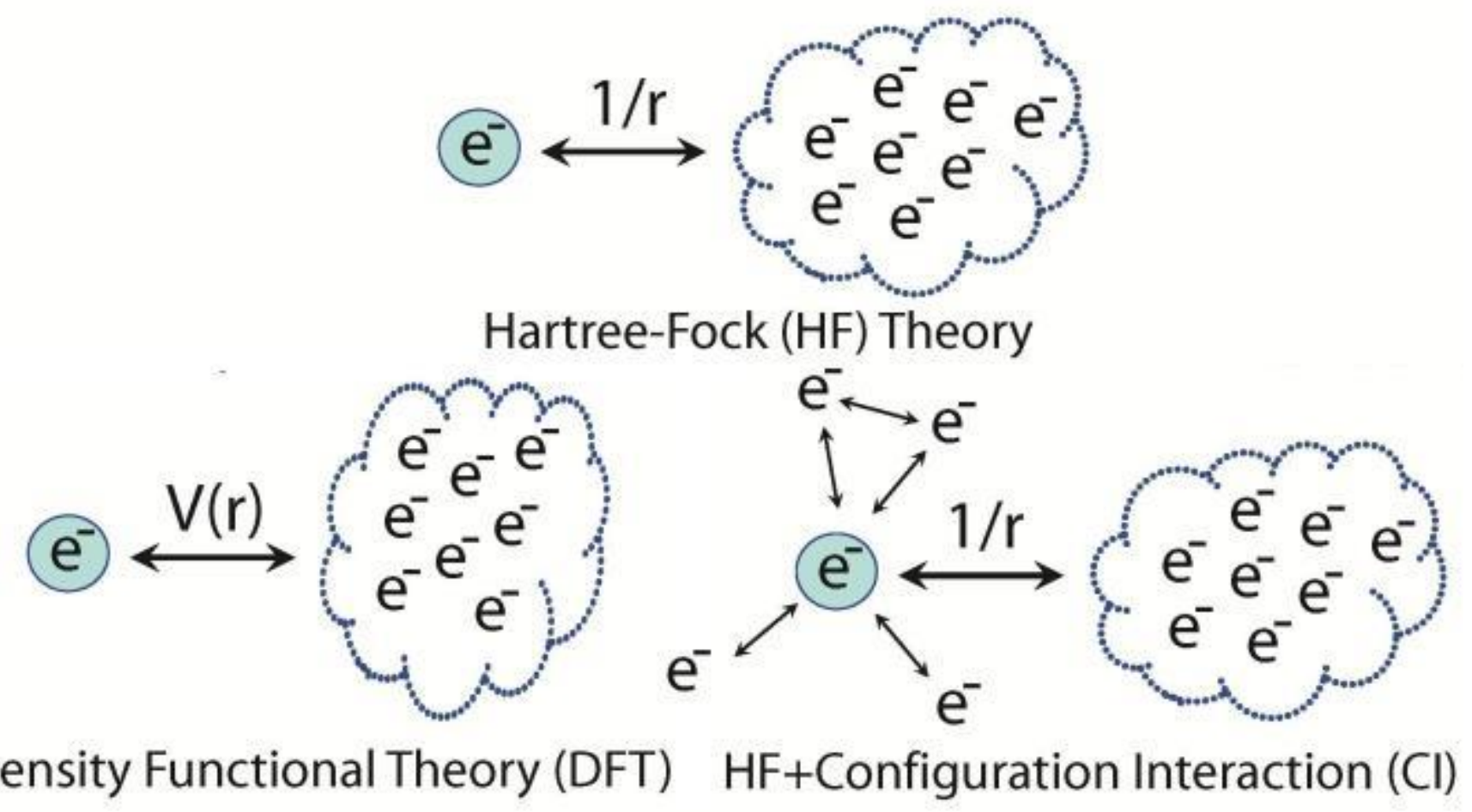




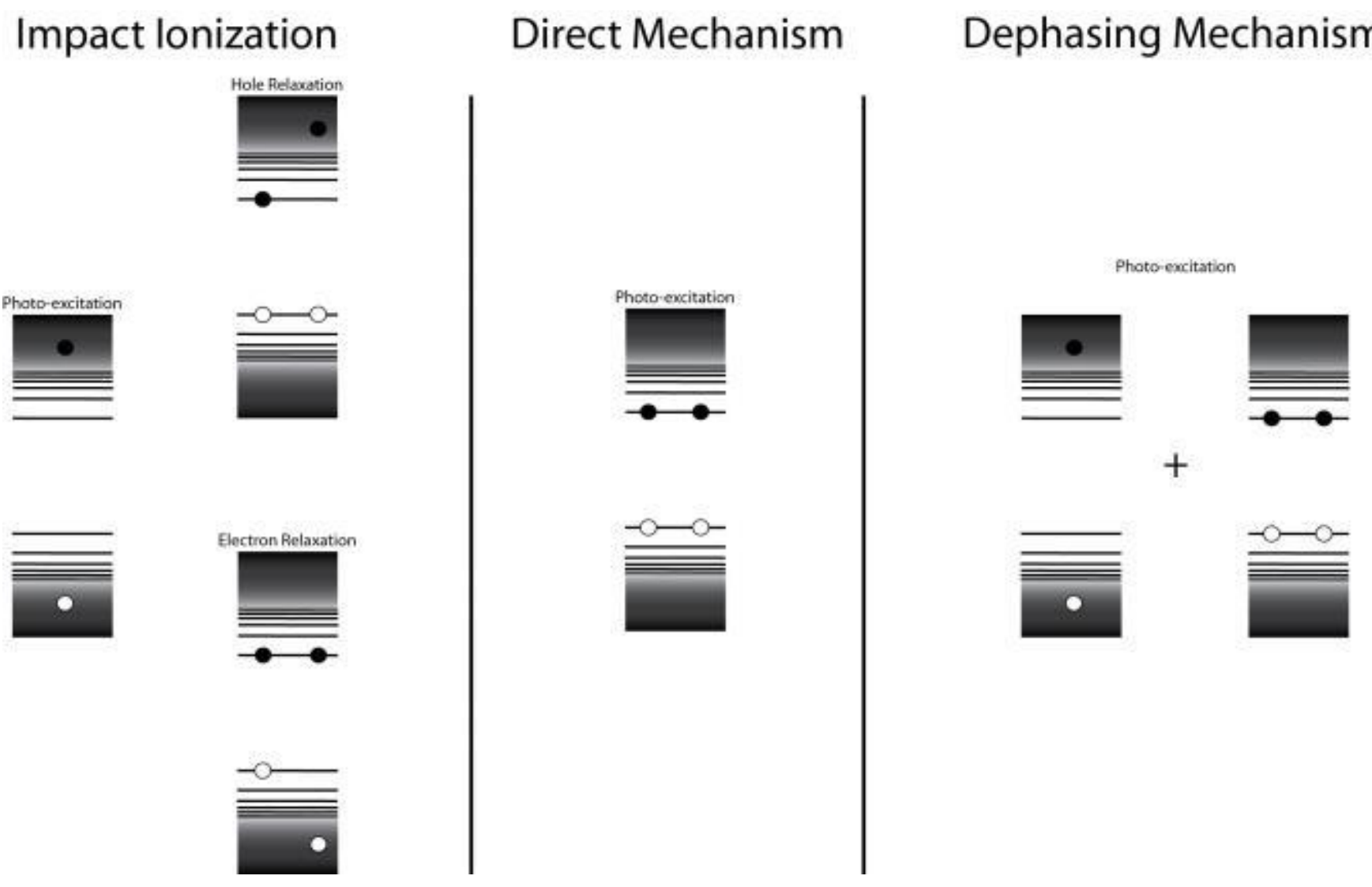




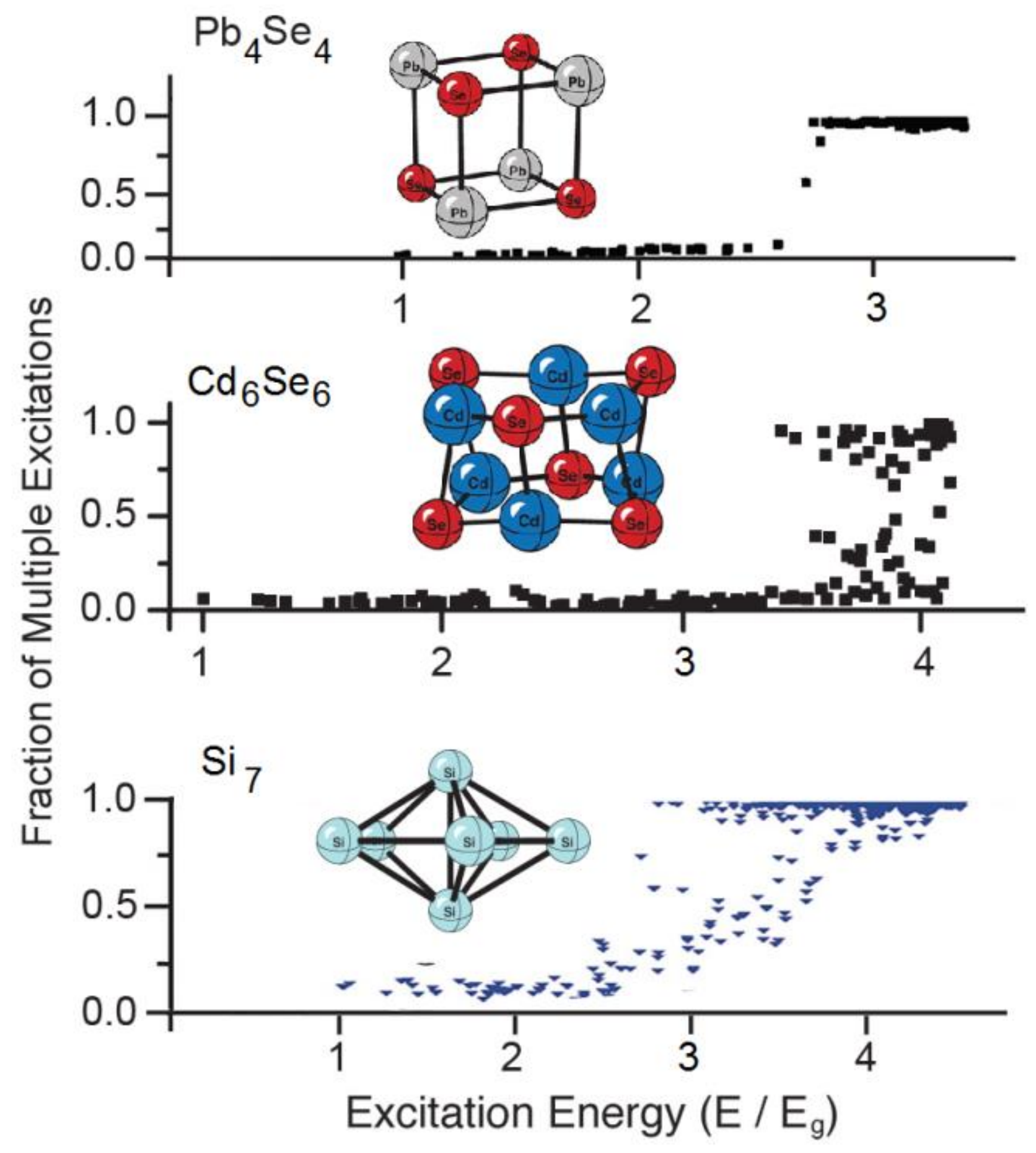




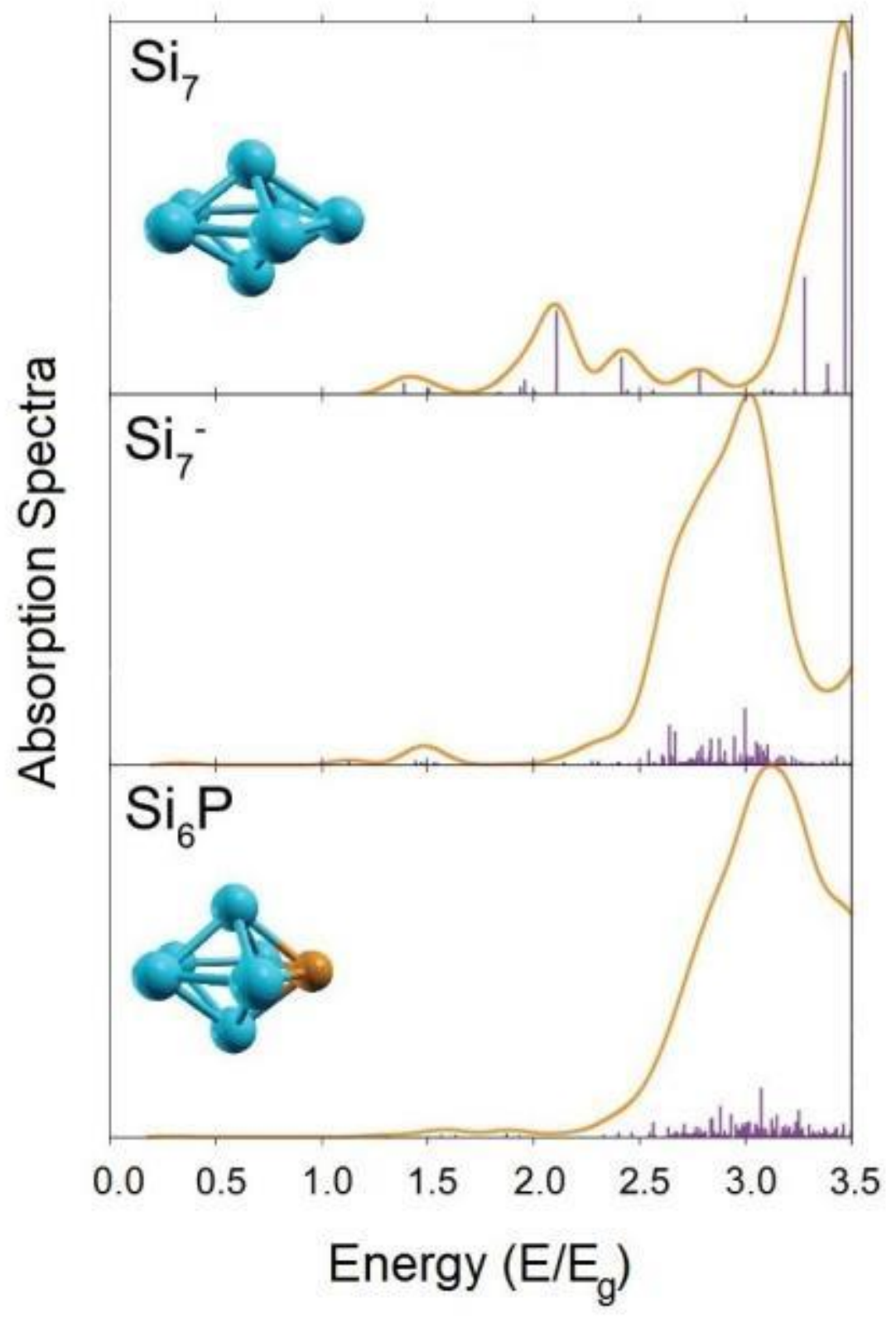



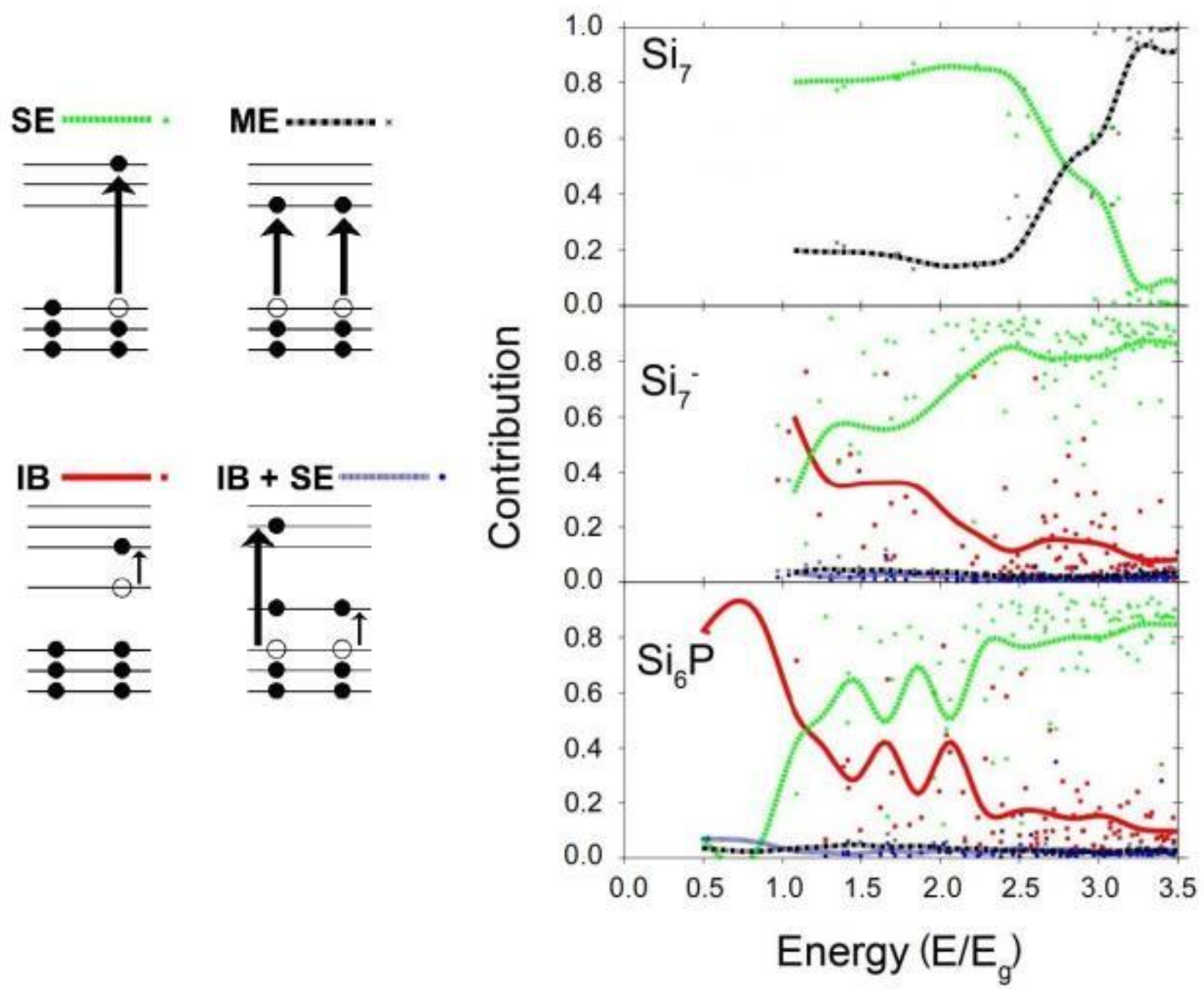

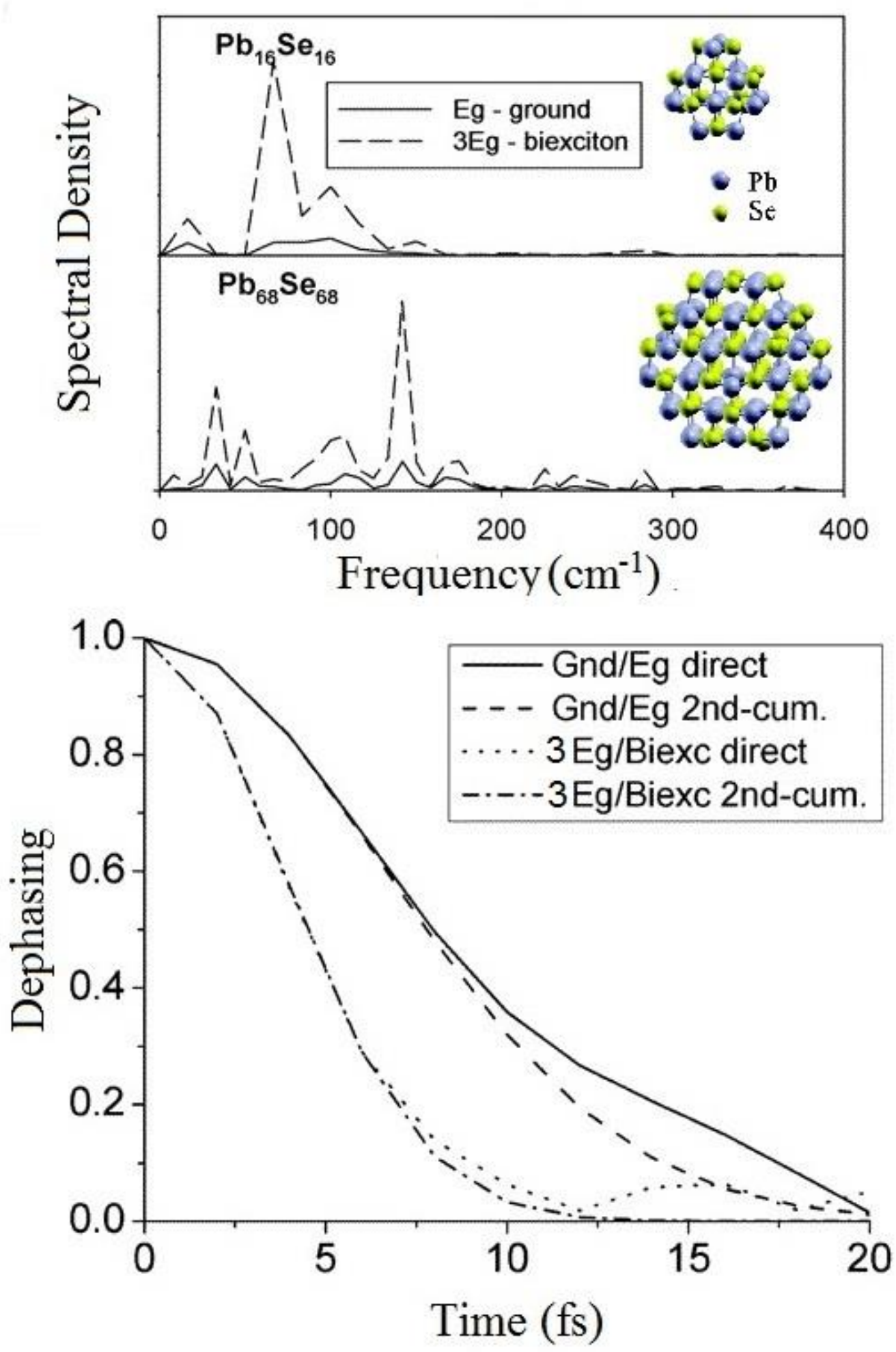

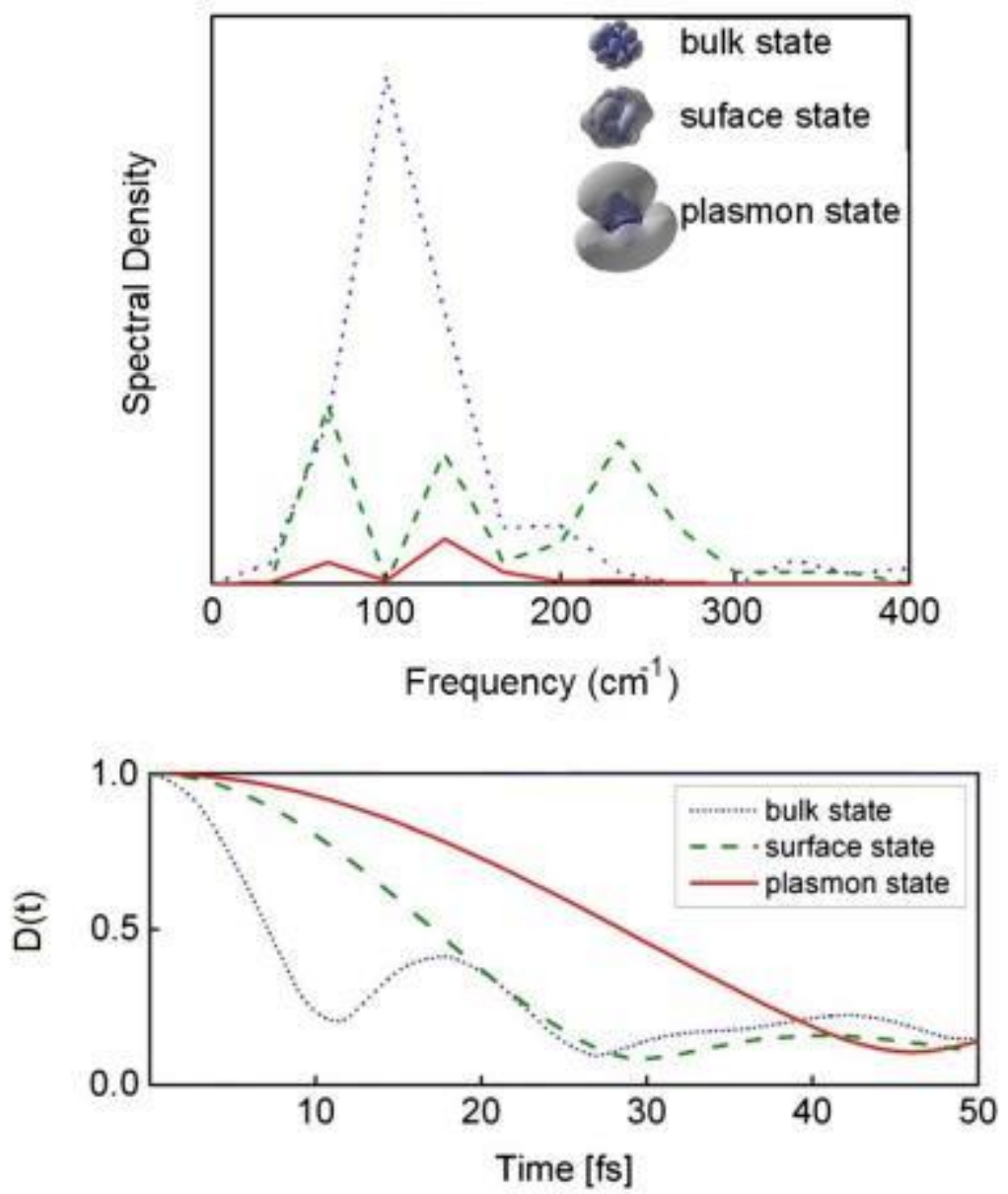


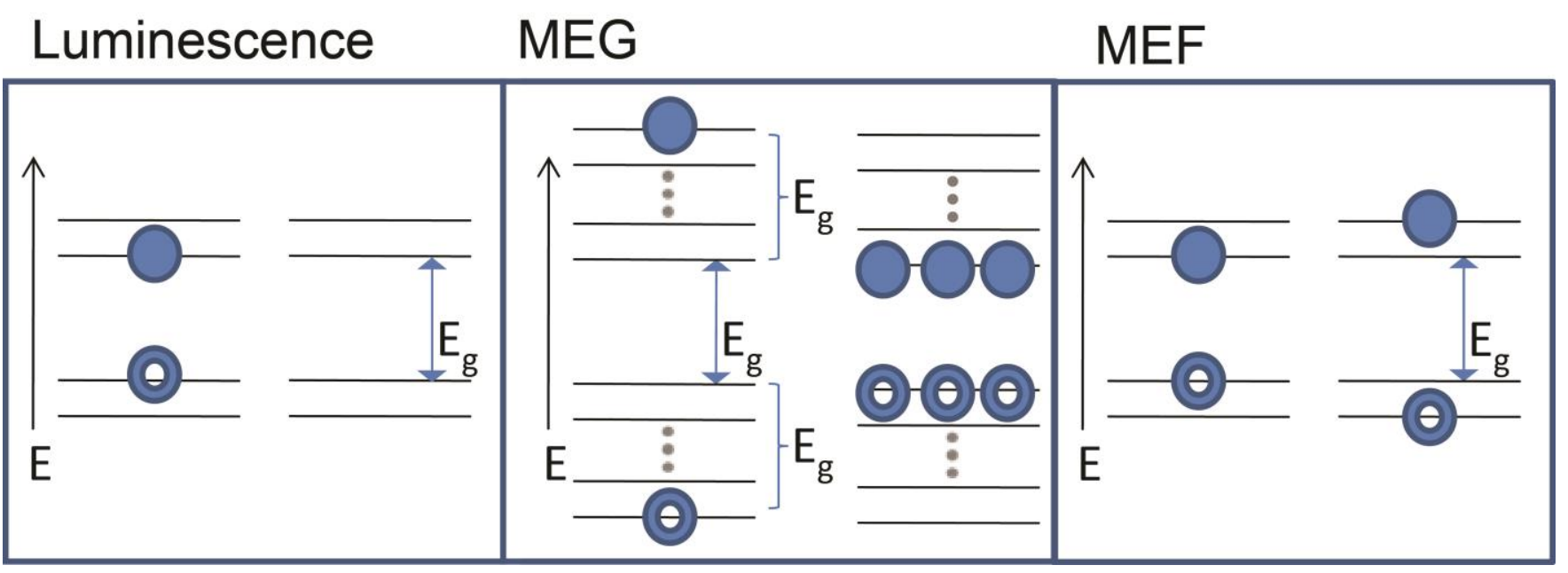



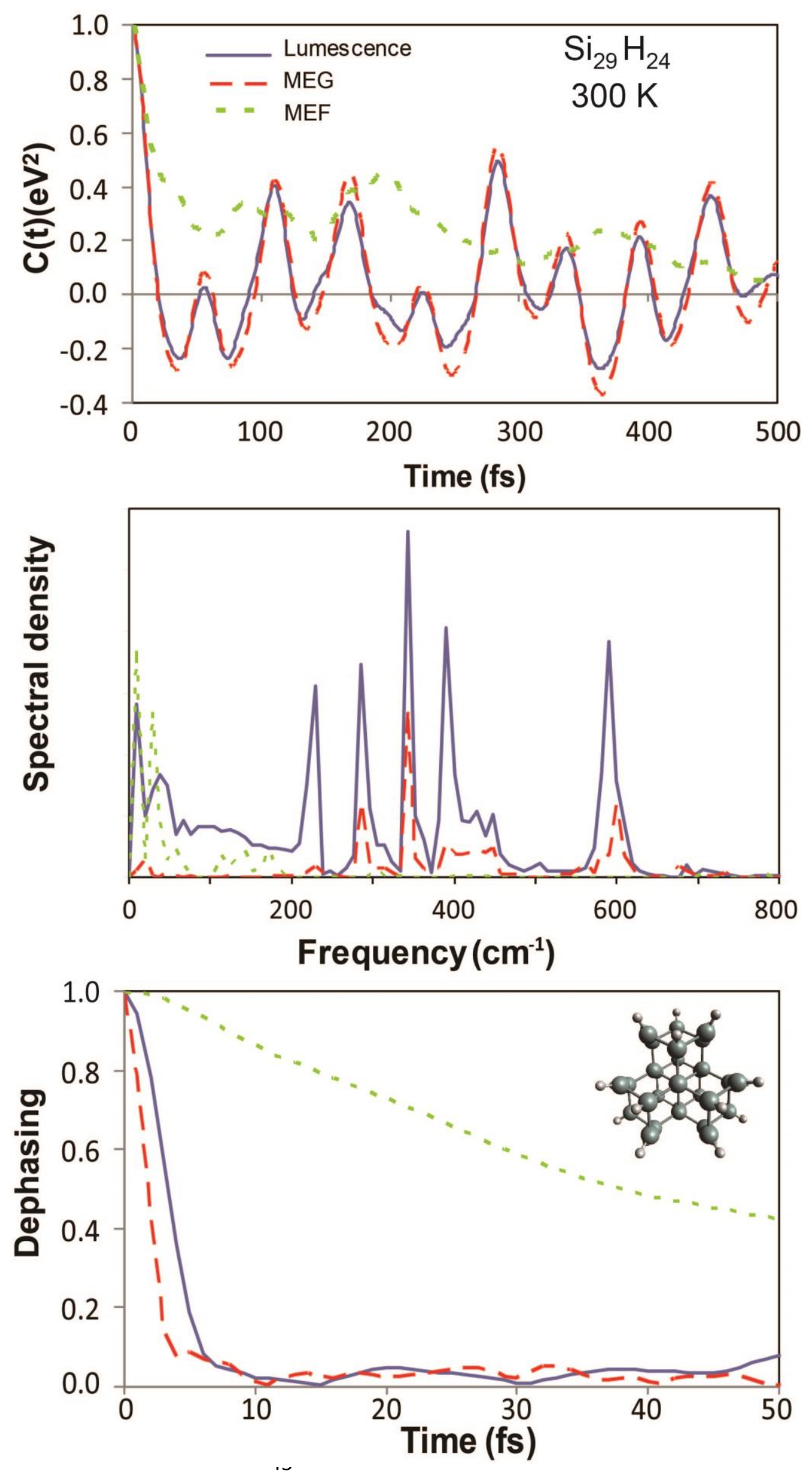

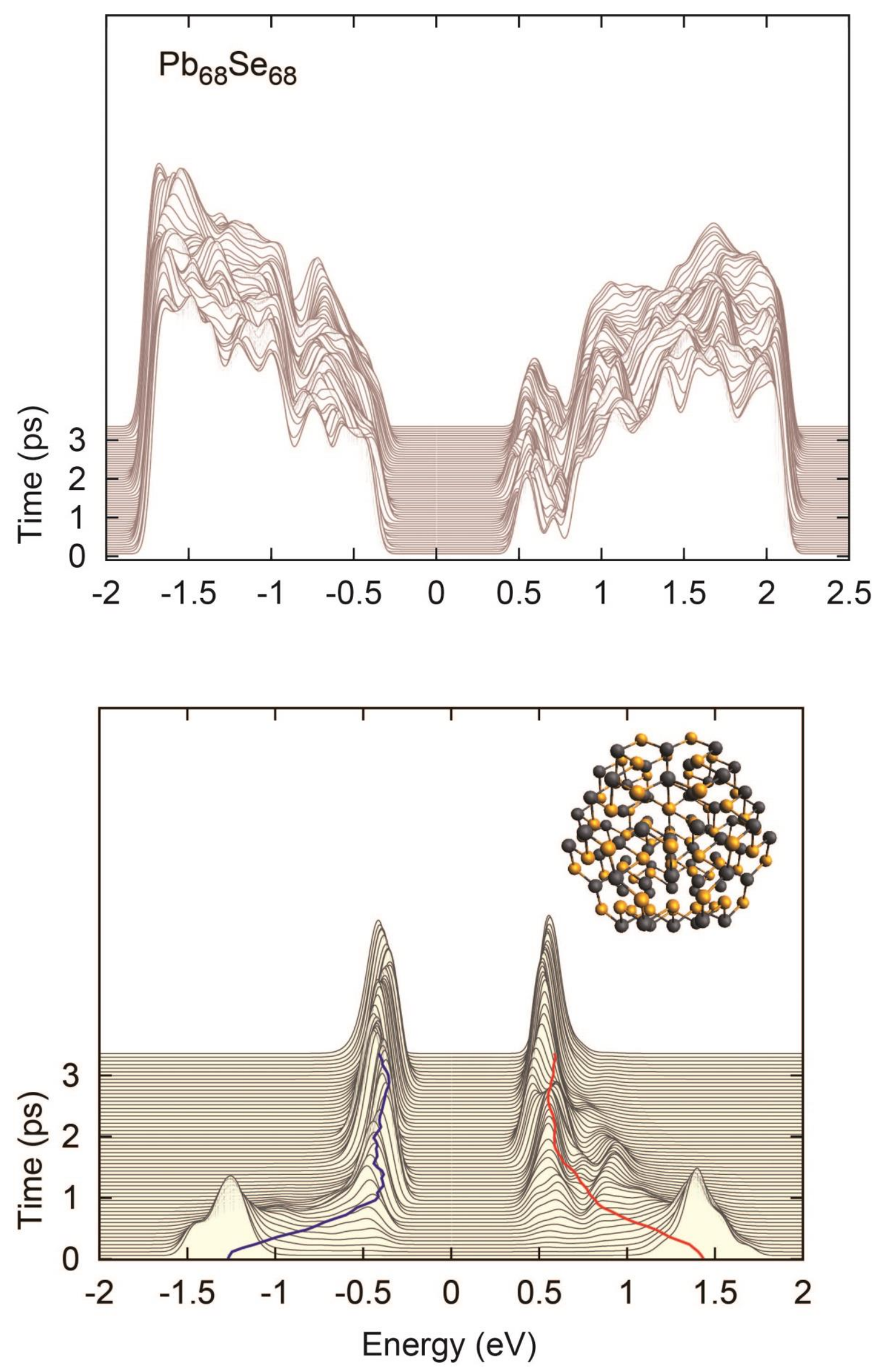


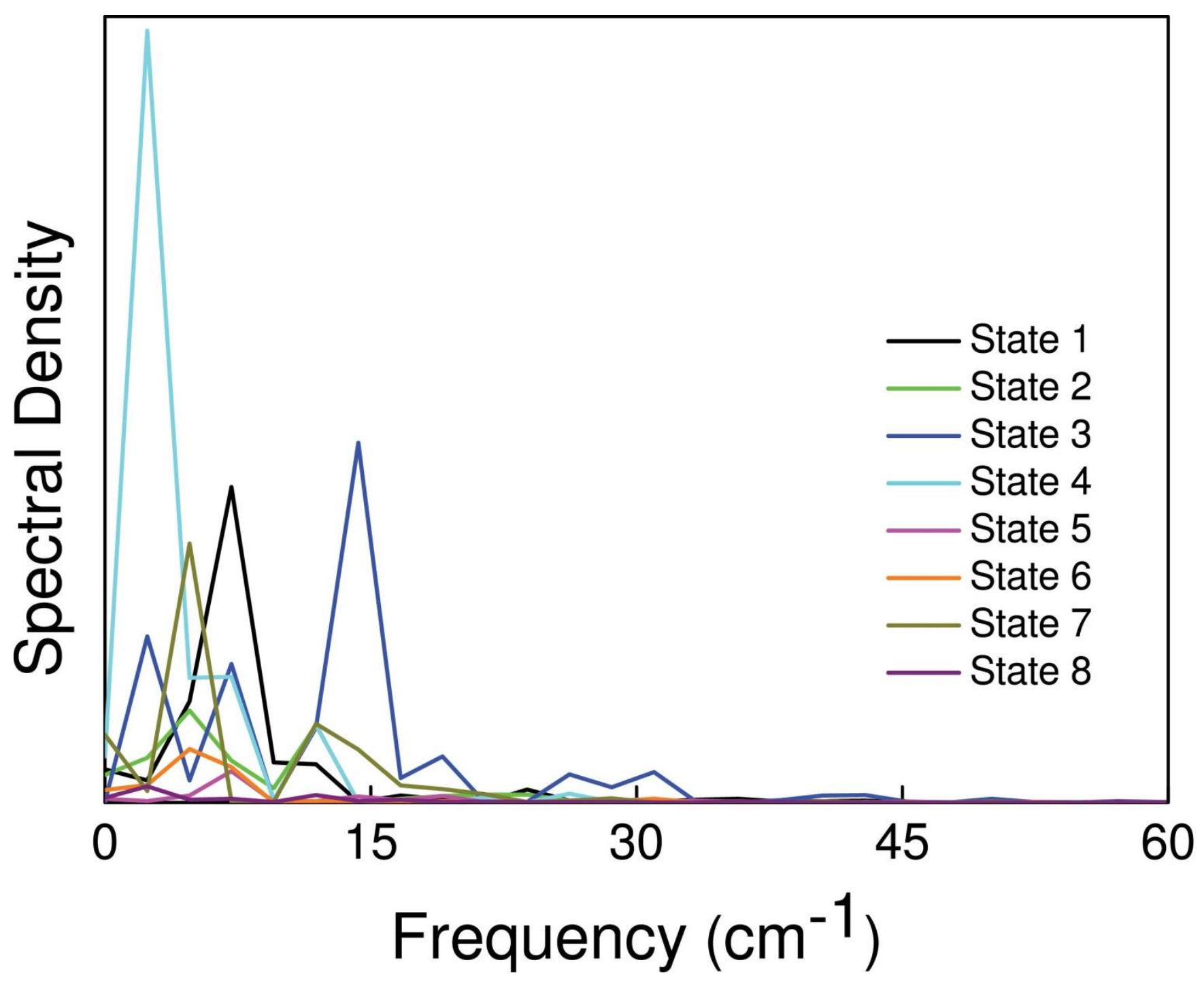




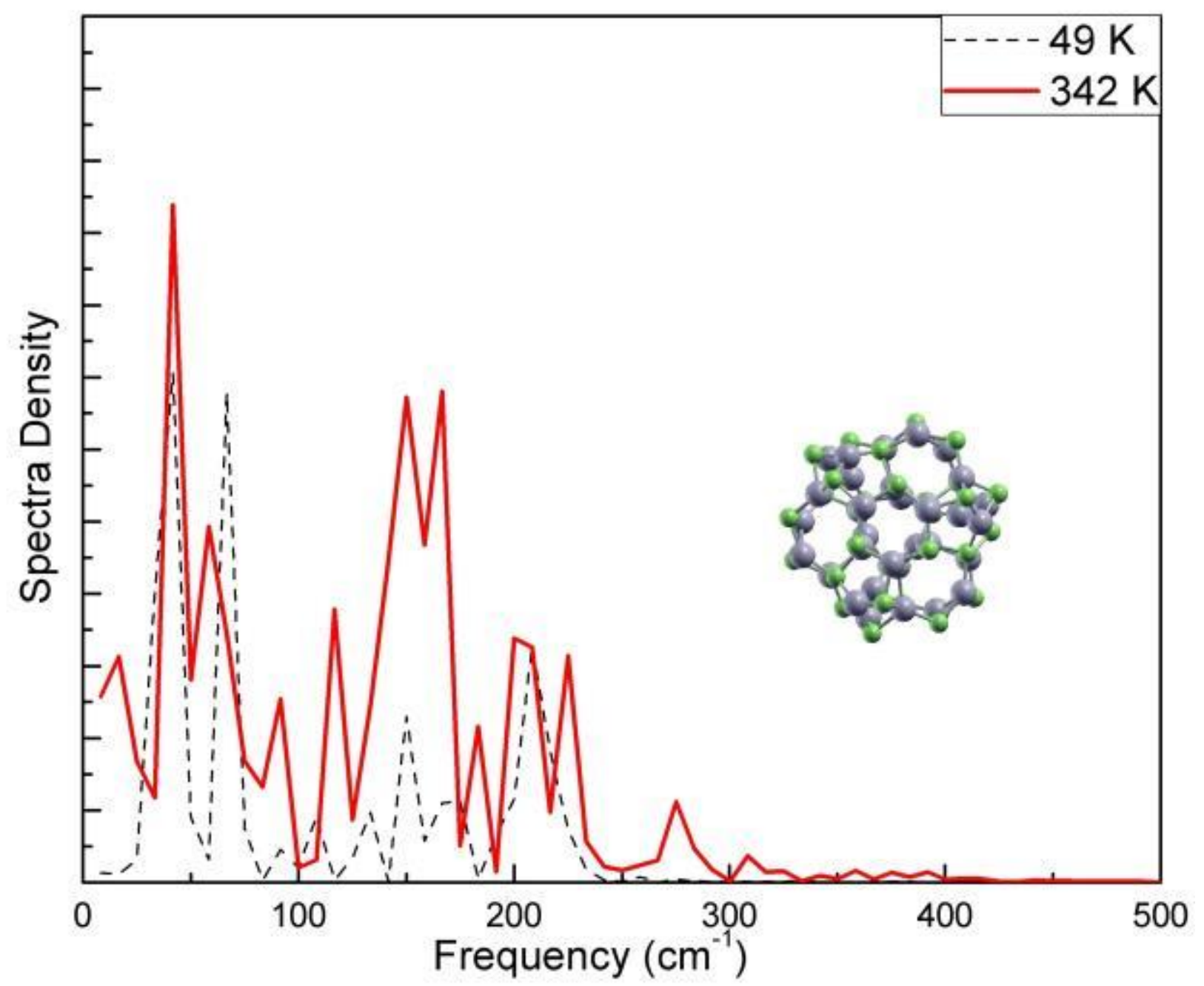




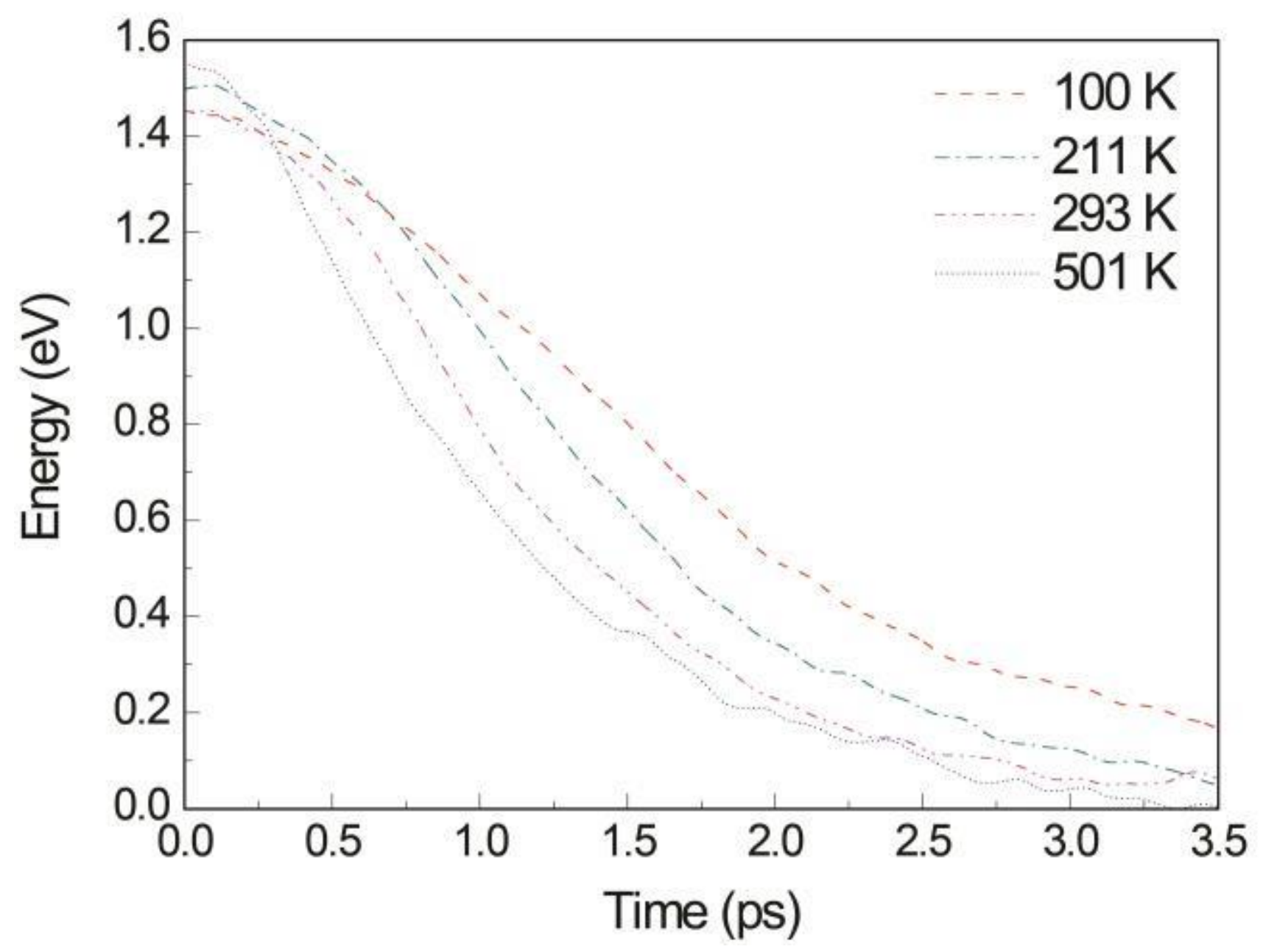




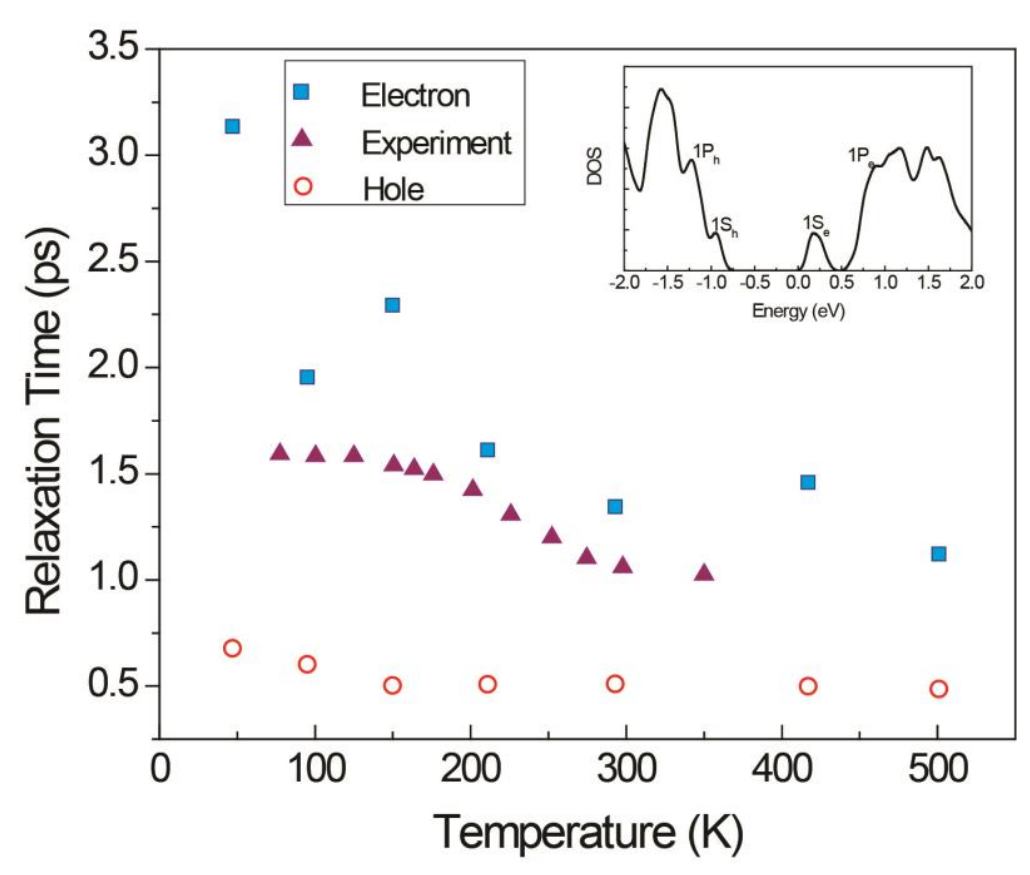



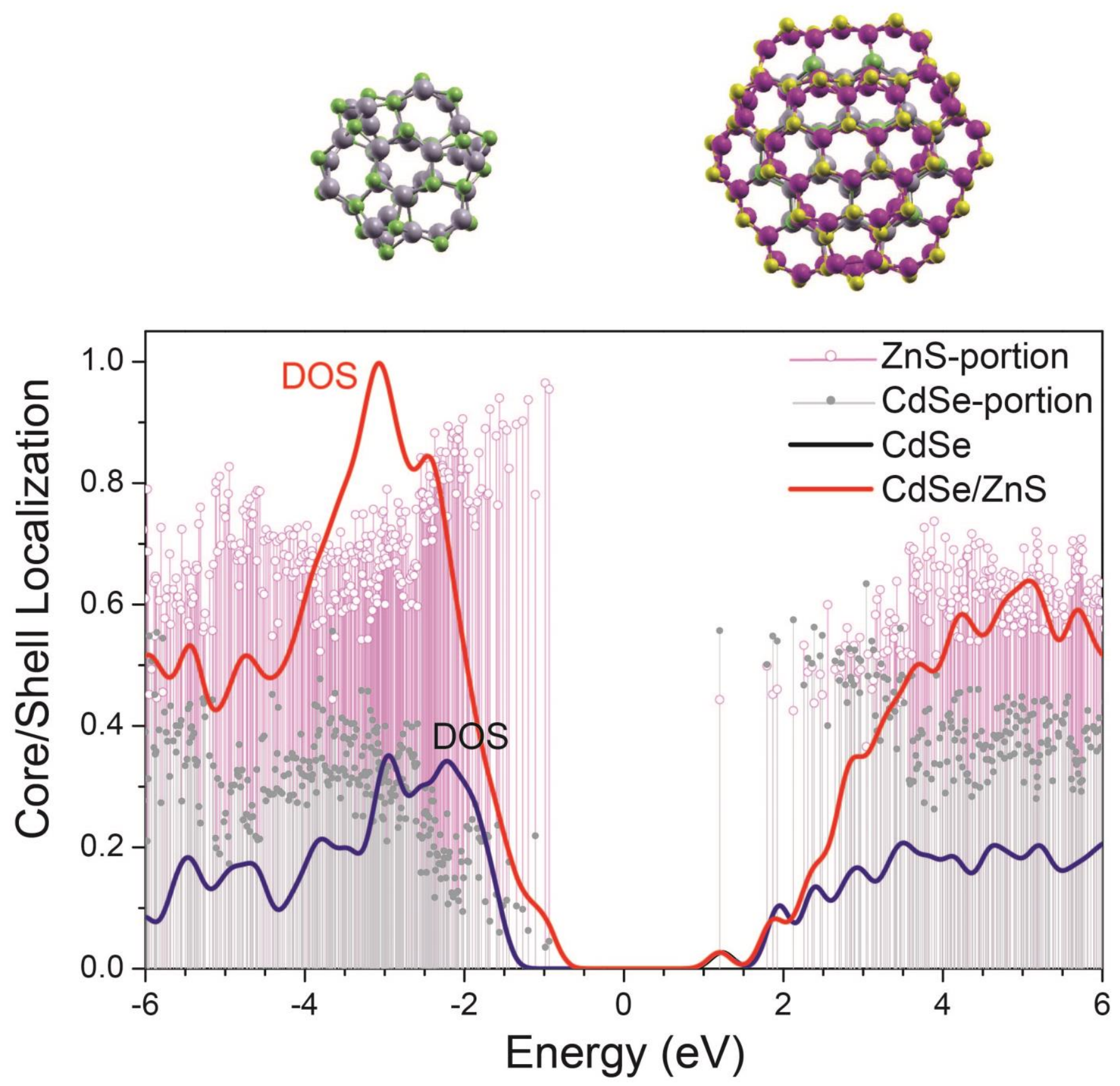


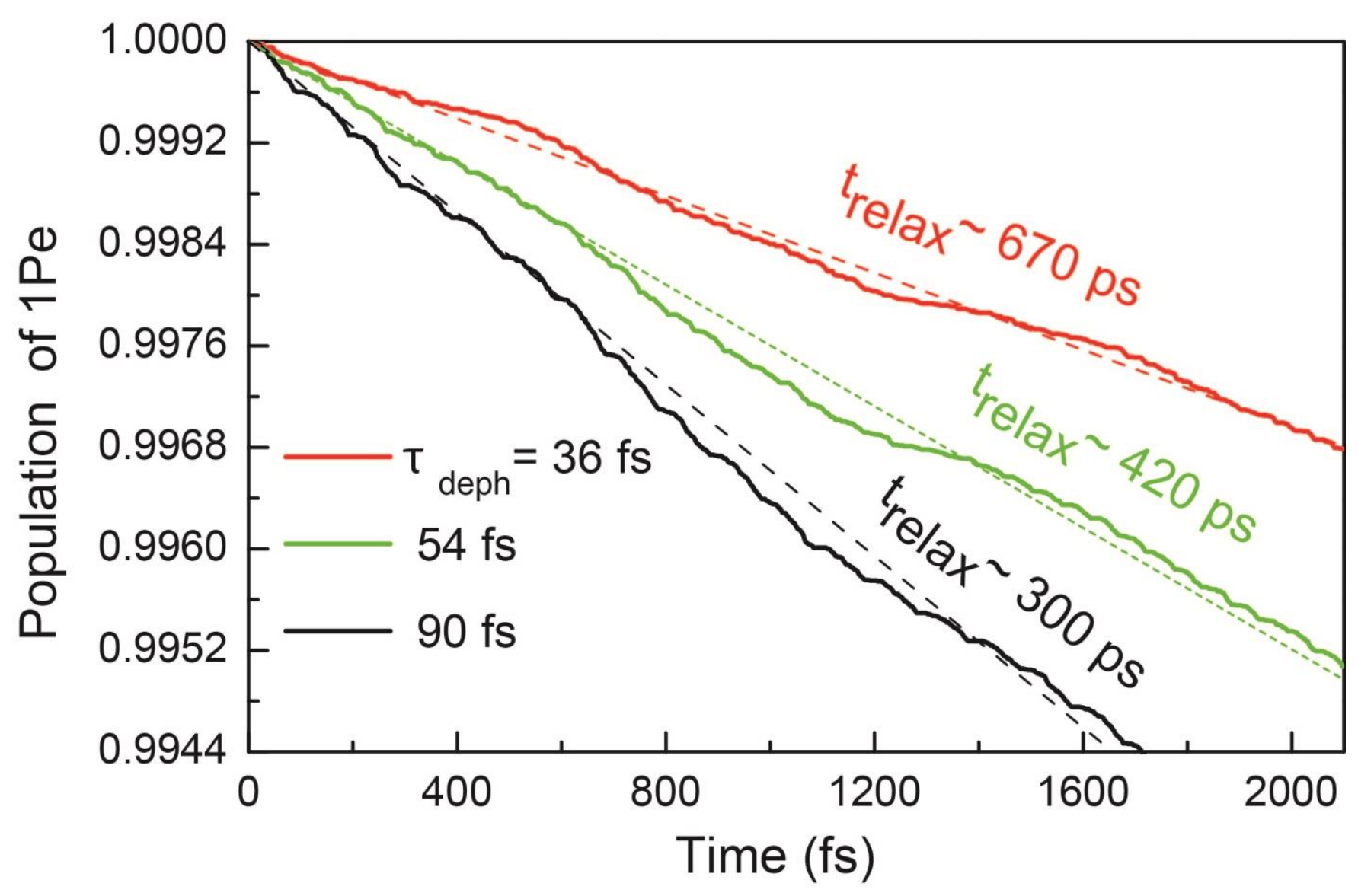



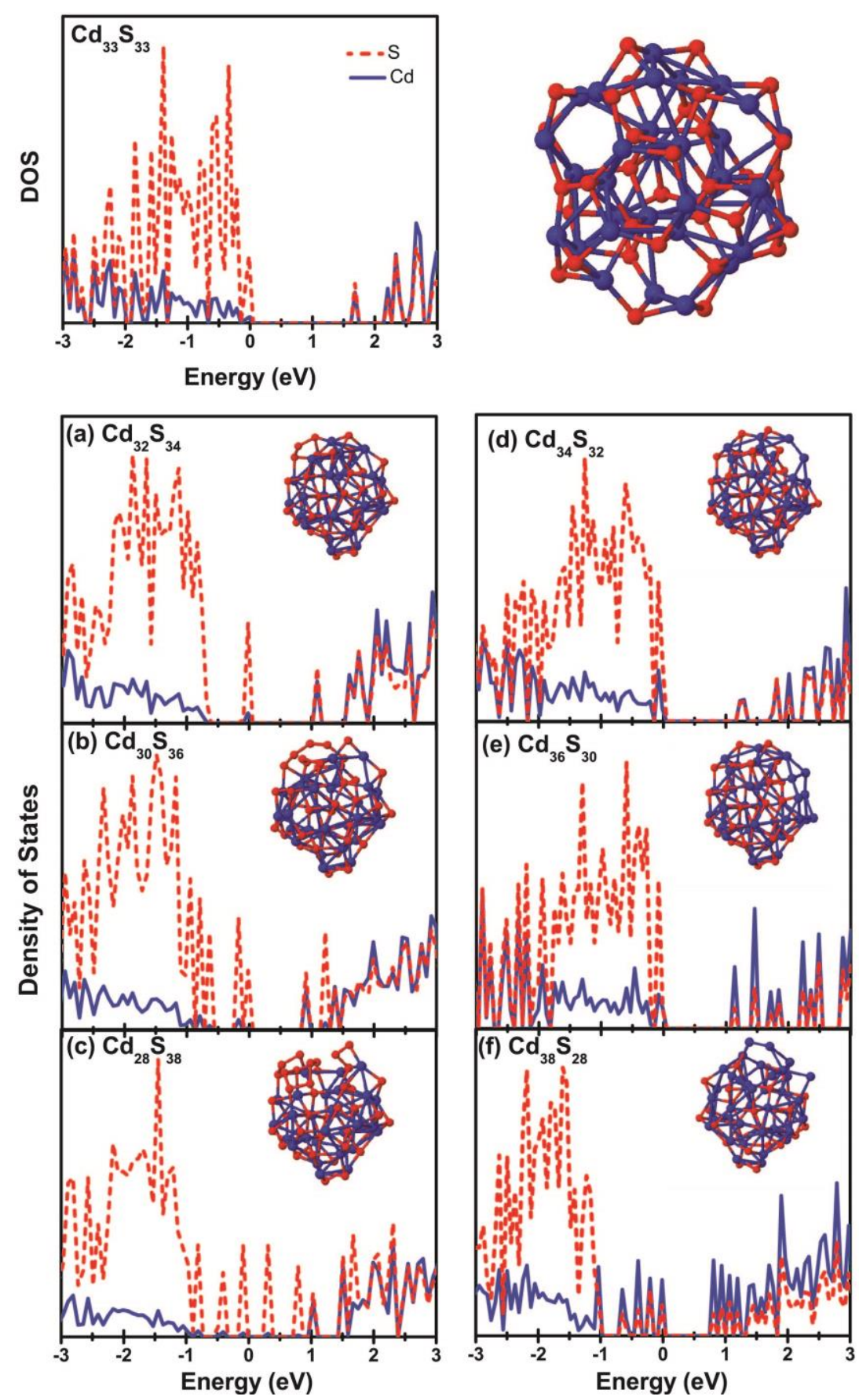

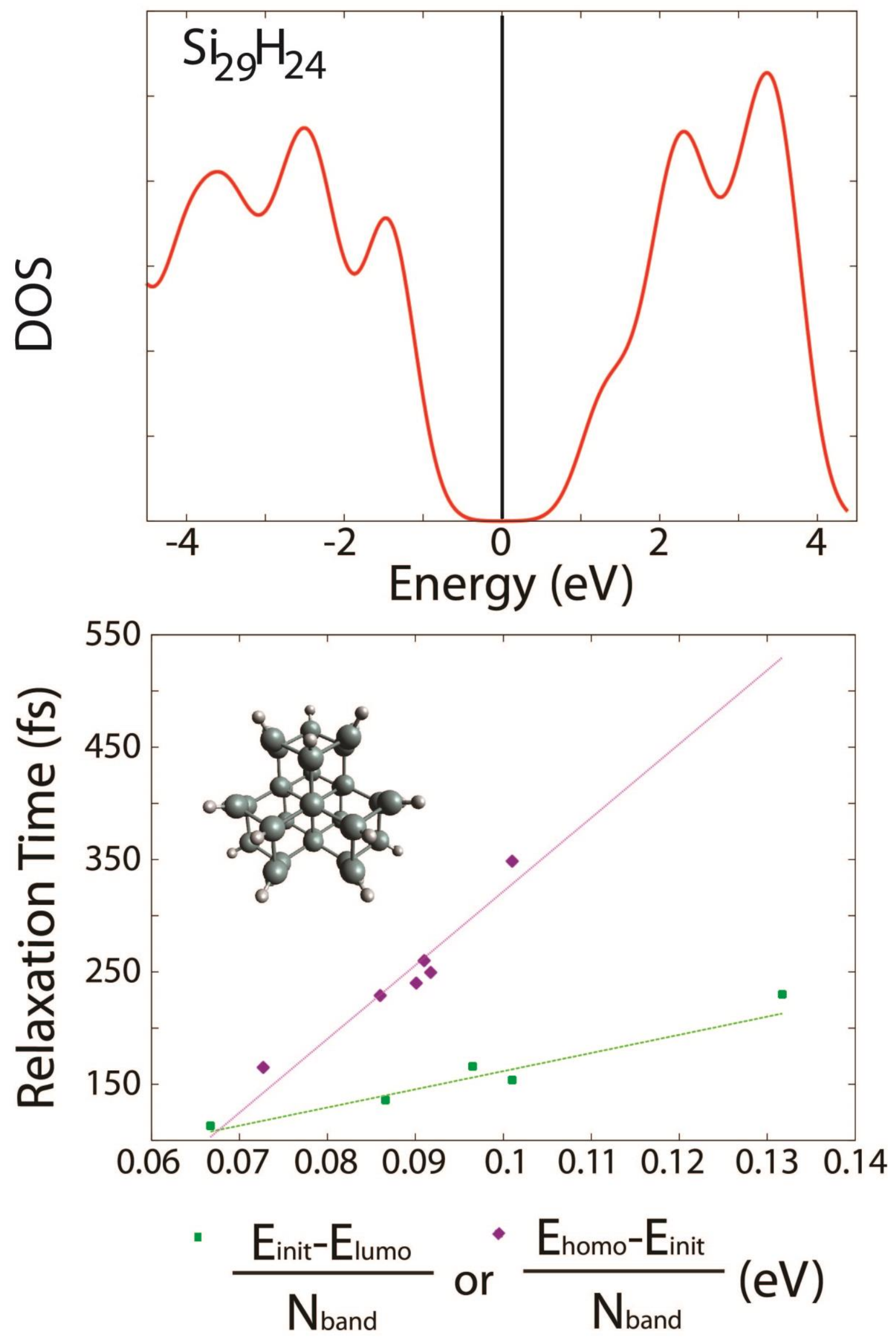

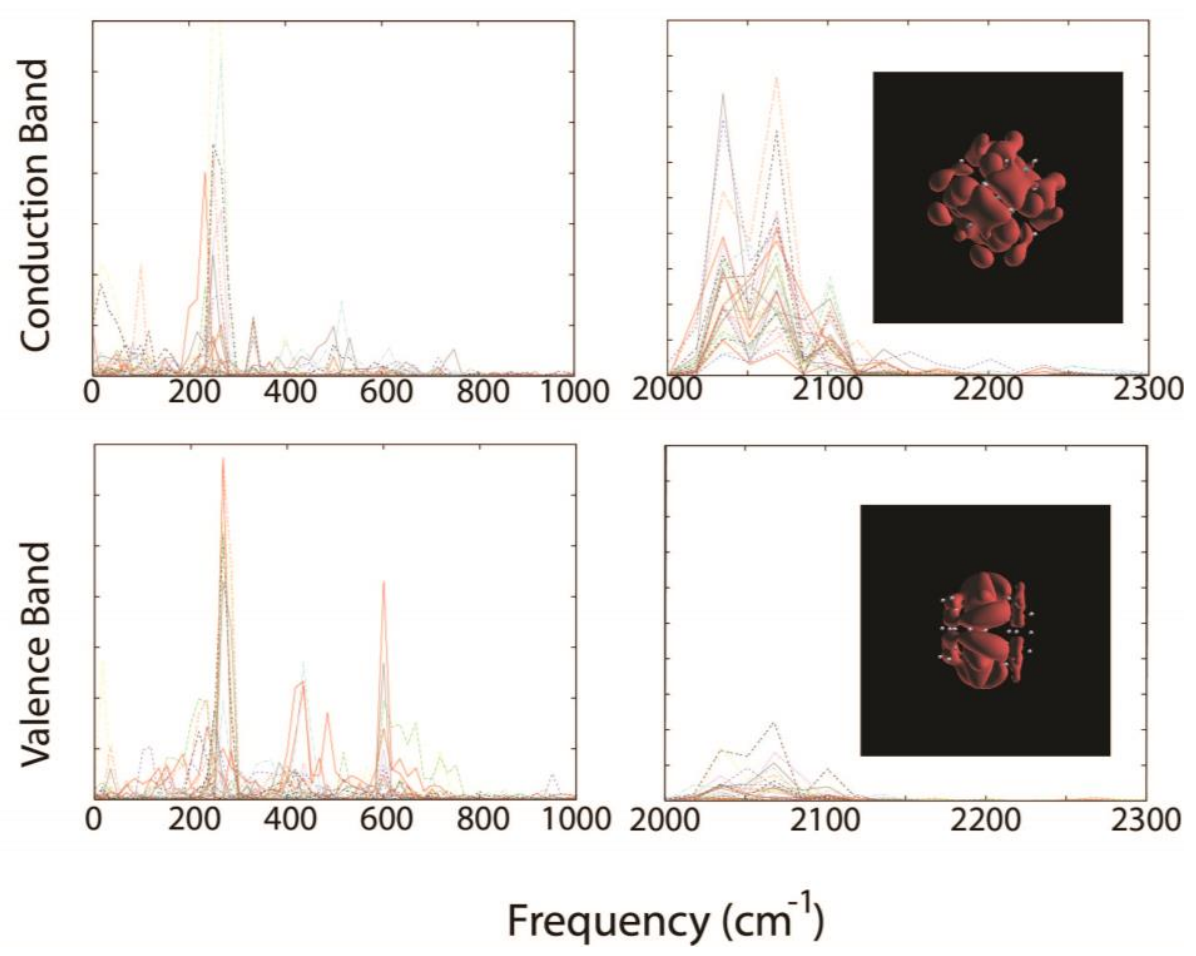


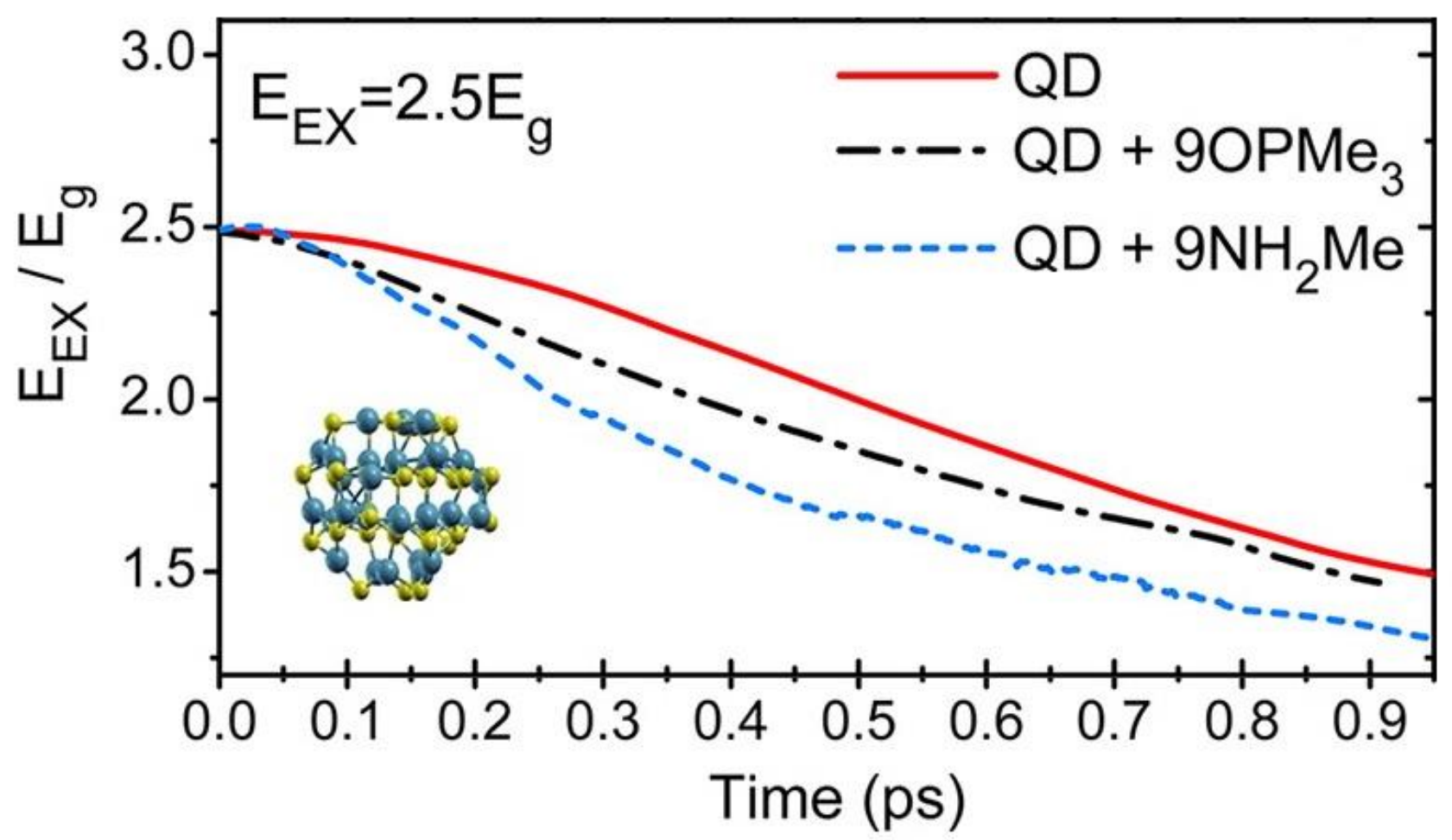

